\title{
On lower semicontinuity of the quantum conditional mutual information and its corollaries
}

\author{
M.E. Shirokov*
}

\begin{abstract}
It is well known that the quantum mutual information and its conditional version do not increase under local channels. I this paper we show that the recently established lower semicontinuity of the quantum conditional mutual information implies (in fact, is equivalent to) the lower semicontinuity of the loss of the quantum (conditional) mutual information under local channels considered as a function on the Cartesian product of the set of all states of a composite system and the sets of all local channels (equipped with the strong convergence).

Some applications of this property are considered. New continuity conditions for the quantum mutual information and for the squashed entanglement in both bipartite and multipartite infinite-dimensional systems are obtained. It is proved, in particular, that the multipartite squashed entanglement of any countably-nondecomposable separable state with finite marginal entropies is equal to zero.

Special continuity properties of the information gain of a quantum measurement with and without quantum side information are established that can be treated as robustness (stability) of these quantities w.r.t. perturbation of the measurement and the measured state.
\end{abstract}

\section{Contents}

1 Introduction

2 Preliminaries

3 Extended QCMI and its properties 6

3.1 Tripartite system . . . . . . . . . . . . . . . . . . 6

3.2 Multipartite system . . . . . . . . . . . . . . . . . . 9

4 Lower semicontinuity of the loss of QCMI under local channels $\quad 10$

4.1 Tripartite system . . . . . . . . . . . . . . . . . 10

4.2 Multipartite system . . . . . . . . . . . . . . . . . . . 13

*Steklov Mathematical Institute, RAS, Moscow, email:msh@mi.ras.ru 
5 Applications 15

5.1 Continuity conditions for QCMI . . . . . . . . . . . . 15

5.1 .1 Tripartite system . . . . . . . . . . . . . . . . 15

5.1 .2 Multipartite system . . . . . . . . . . . . . . . . 17

5.2 Continuity conditions for the squashed entanglement . . . . . . 18

5.2 .1 Bipartite system . . . . . . . . . . . . . . . . 18

5.2 .2 Multipartite system . . . . . . . . . . . . . . . 20

5.3 On robustness of information gain of quantum measurements . . . . . 26

\section{Introduction}

The quantum mutual information and its conditional version are basic entropic quantities essentially used in different tasks of quantum information theory [15, 25, 38. In finite dimensions these quantities are continuous bounded functions on the set of all states of a composite quantum system. In the case of bipartite system $A B$ and conditioning system $C$ they are defined by the expressions

$$
I(A: B)_{\rho}=H\left(\rho_{A}\right)+H\left(\rho_{B}\right)-H\left(\rho_{A B}\right)
$$

and

$$
I(A: B \mid C)_{\rho}=H\left(\rho_{A C}\right)+H\left(\rho_{B C}\right)-H\left(\rho_{A B C}\right)-H\left(\rho_{C}\right) .
$$

In the infinite-dimensional case these formulae may contain the uncertainty $+\infty-\infty$. So, in this case one should use expressions for $I(A: B)_{\rho}$ and $I(A: B \mid C)_{\rho}$ which are less sensitive to infinite values of marginal entropies.

It is well known that the quantum mutual information has a nonnegative lower semicontinuous extension to the set of all states of infinite-dimensional composite system. In the bipartite case this extension is given by the formula

$$
I(A: B)_{\rho}=H\left(\rho_{A B} \| \rho_{A} \otimes \rho_{B}\right),
$$

where $H(\cdot \| \cdot)$ is the quantum relative entropy, coinciding with (11) for a state $\rho_{A B}$ with finite marginal entropies $H\left(\rho_{A}\right)$ and $H\left(\rho_{B}\right)$ [23].

For the quantum conditional mutual information (QCMI) there are several expressions permitting to define it for states with infinite marginal entropies. For example, in the tripartite case we have

$$
I(A: B \mid C)_{\rho}=I(A C: B)_{\rho}-I(B: C)_{\rho} \quad \text { and } \quad I(A: B \mid C)_{\rho}=I(A: B C)_{\rho}-I(A: C)_{\rho} .
$$

But these and similar expressions (see [29, Section 6]) are not well defined on whole space of states of an infinite-dimensional composite system.

Nevertheless, it is shown in 29] that in both tripartite and multipartite cases there exists a unique lower semicontinuous function well defined on the whole space of states of an infinite-dimensional composite system possessing all basic properties of QCMI 
(in particular, coinciding with the original definition (2) and all alternative expressions for QCMI at states, where they are well defined). Unfortunately, this function can not be expressed by a simple formula, it is defined via some optimization procedure (see details in Section 3).

Despite the absent of explicit formula, the existence of a unique extension of QCMI to the whole space of states of an infinite-dimensional composite system is important because it allows to not care about the characteristics of the state at which QCMI is calculated in formal manipulations. For example, when we apply a quantum channel to a part of a composite state at which QCMI is well defined by a simple formula we may obtain a state at which this formula does not work, but the existence of global extension of QCMI allows to forget about this problem. By this reason the global extension of QCMI plays a central role in the resent works [31, 32], where the main results are formulated as a property valid for any channel between given quantum systems: the uniform continuity of basic capacities on the set of all channels, the uniform finitedimensional approximations of basic capacities, etc. The global extension of QCMI is also used essentially in the recent work [9].

In this paper we focus attention on the lower semicontinuity of the global extension of QCMI mentioned before on the whole space of states of an infinite-dimensional composite system. In the tripartite case it means that

$$
\liminf _{n \rightarrow+\infty} I(A: B \mid C)_{\rho^{n}} \geq I(A: B \mid C)_{\rho^{0}}
$$

for any sequence of states $\rho_{A B C}^{n}$ converging to a state $\rho_{A B C}^{0}$. It is equivalent to the closedness of the set of all states $\rho_{A B C}$ determined by the inequality

$$
I(A: B \mid C)_{\rho} \leq \epsilon
$$

for any nonnegative $\epsilon$. For $\epsilon=0$ this means the closedness of the set of short Markov chains [13], which by no means is obvious in infinite dimensions.

By using the lower semicontinuity of QCMI and the Stinespring representation of strongly converging sequences of quantum channels obtained in [33] we prove (in Section 4) the lower semicontinuity of the loss of the quantum (conditional) mutual information under action of local channels considered as a function on the Cartesian product of the set of all states of a composite system and the sets of all local channels (equipped with the strong convergence topology). In the tripartite case it means that the function

$$
\left(\rho, \Phi_{A}, \Phi_{B}\right) \mapsto I(A: B \mid C)_{\rho}-I\left(A^{\prime}: B^{\prime} \mid C\right)_{\Phi_{A} \otimes \Phi_{B} \otimes \operatorname{Id}_{C}(\rho)},
$$

where $\Phi_{X}$ denotes a channel from $X$ to $X^{\prime}$, is lower semicontinuous on the set of all triplets $\left(\rho_{A B C}, \Phi_{A}, \Phi_{B}\right)$ such that $I\left(A^{\prime}: B^{\prime} \mid C\right)_{\Phi_{A} \otimes \Phi_{B} \otimes \operatorname{Id}_{C}(\rho)}<+\infty$ w.r.t. the convergence:

$\left\{\left(\rho_{A B C}^{n}, \Phi_{A}^{n}, \Phi_{B}^{n}\right) \rightarrow\left(\rho_{A B C}^{0}, \Phi_{A}^{0}, \Phi_{B}^{0}\right)\right\} \Leftrightarrow\left\{\rho_{A B C}^{n} \rightarrow \rho_{A B C}^{0}\right\} \wedge\left\{\Phi_{A}^{n} \rightarrow \Phi_{A}^{0}\right\} \wedge\left\{\Phi_{B}^{n} \rightarrow \Phi_{B}^{0}\right\}$, where $\Phi_{X}^{n} \rightarrow \Phi_{X}^{0}$ denotes the strong convergence of the sequence $\left\{\Phi_{X}^{n}\right\}$ to the channel $\Phi_{X}^{0}$ which means that $\left\{\Phi_{X}^{n}\left(\varrho_{X}\right)\right\}$ tends to the state $\Phi_{X}^{0}\left(\varrho_{X}\right)$ for any state $\varrho_{X}$. 
The above property has a direct analogy to the lower semicontinuity of the entropic disturbance 1 as function of a pair (channel, input ensemble) established in [33]. In fact, if we restrict attention to discrete (finite or countable) ensembles of quantum states then the lower semicontinuity of the entropic disturbance can be derived from the lower semicontinuity of the loss of the quantum mutual information under action of a local channel.

The lower semicontinuity of the loss of QCMI under local channels allows to show that the action of arbitrary local channels preserves continuity of QCMI. In the tripartite case it means that the limit relation

$$
\lim _{n \rightarrow+\infty} I(A: B \mid C)_{\rho^{n}}=I(A: B \mid C)_{\rho^{0}}<+\infty
$$

for some sequence of states $\rho_{A B C}^{n}$ converging to a state $\rho_{A B C}^{0}$ implies that

$$
\lim _{n \rightarrow+\infty} I\left(A^{\prime}: B^{\prime} \mid C\right)_{\Phi_{A} \otimes \Phi_{B} \otimes \operatorname{Id}_{C}\left(\rho^{n}\right)}=I\left(A^{\prime}: B^{\prime} \mid C\right)_{\Phi_{A} \otimes \Phi_{B} \otimes \operatorname{Id}_{C}\left(\rho^{0}\right)}
$$

for arbitrary channels $\Phi_{A}: A \rightarrow A^{\prime}$ and $\Phi_{B}: B \rightarrow B^{\prime}$. Moreover, this result can be strengthened by replacing fixed channels $\Phi_{A}$ and $\Phi_{B}$ by strongly converging sequences of channels (see Proposition 2 in Section 5.1.1)

In Section 5 we show how the above "preserving continuity" property can be used in continuity analysis of QCMI and relating important characteristics of quantum states and quantum measurements.

\section{Preliminaries}

Let $\mathcal{H}$ be a separable Hilbert space, $\mathfrak{B}(\mathcal{H})$ the algebra of all bounded operators on $\mathcal{H}$ with the operator norm $\|\cdot\|$ and $\mathfrak{T}(\mathcal{H})$ the Banach space of all trace-class operators on $\mathcal{H}$ with the trace norm $\|\cdot\|_{1}$. Let $\mathfrak{S}(\mathcal{H})$ be the set of quantum states (positive operators in $\mathfrak{T}(\mathcal{H})$ with unit trace) [15, 25, 38].

Denote by $I_{\mathcal{H}}$ the unit operator on a Hilbert space $\mathcal{H}$ and by $\operatorname{Id}_{\mathcal{H}}$ the identity transformation of the Banach space $\mathfrak{T}(\mathcal{H})$.

The von Neumann entropy of a quantum state $\rho \in \mathfrak{S}(\mathcal{H})$ is defined by the formula $H(\rho)=\operatorname{Tr} \eta(\rho)$, where $\eta(x)=-x \log x$ for $x>0$ and $\eta(0)=0$. It is a concave lower semicontinuous function on the set $\mathfrak{S}(\mathcal{H})$ taking values in $[0,+\infty]$ [15, 24, 36]. The von Neumann entropy satisfies the inequality

$$
H(p \rho+(1-p) \sigma) \leq p H(\rho)+(1-p) H(\sigma)+h_{2}(p)
$$

valid for any states $\rho$ and $\sigma$ in $\mathfrak{S}(\mathcal{H})$ and $p \in(0,1)$, where $h_{2}(p)=\eta(p)+\eta(1-p)$ is the binary entropy [25, 38].

\footnotetext{
${ }^{1}$ The entropic disturbance is the difference between the Holevo quantities of an ensemble of quantum states and the image of this ensemble under a quantum channel [3, 5].
} 
The quantum relative entropy for two states $\rho$ and $\sigma$ in $\mathfrak{S}(\mathcal{H})$ is defined as

$$
H(\rho \| \sigma)=\sum\langle i|\rho \log \rho-\rho \log \sigma| i\rangle,
$$

where $\{|i\rangle\}$ is the orthonormal basis of eigenvectors of the state $\rho$ and it is assumed that $H(\rho \| \sigma)=+\infty$ if $\operatorname{supp} \rho$ is not contained in $\operatorname{supp} \sigma[15,24,2$

The quantum conditional entropy

$$
H(A \mid B)_{\rho}=H\left(\rho_{A B}\right)-H\left(\rho_{B}\right)
$$

of a state $\rho_{A B}$ with finite marginal entropies is essentially used in analysis of quantum systems [15, 38]. It can be extended to the set of all states $\rho_{A B}$ with finite $H\left(\rho_{A}\right)$ by the formula

$$
H(A \mid B)=H\left(\rho_{A}\right)-H\left(\rho_{A B} \| \rho_{A} \otimes \rho_{B}\right) .
$$

This extension possesses all basic properties of the quantum conditional entropy valid in finite dimensions [19].

The quantum mutual information of a state $\rho_{A B}$ is defined as

$$
I(A: B)_{\rho}=H\left(\rho_{A B} \| \rho_{A} \otimes \rho_{B}\right)=H\left(\rho_{A}\right)+H\left(\rho_{B}\right)-H\left(\rho_{A B}\right),
$$

where the second formula is valid if $H\left(\rho_{A B}\right)$ is finite [23]. Basic properties of the relative entropy show that $\rho_{A B} \mapsto I(A: B)_{\rho}$ is a lower semicontinuous function on the set $\mathfrak{S}\left(\mathcal{H}_{A B}\right)$ taking values in $[0,+\infty]$. It is well known that

$$
I(A: B)_{\rho} \leq 2 \min \left\{H\left(\rho_{A}\right), H\left(\rho_{B}\right)\right\}
$$

for any state $\rho_{A B}[23,38$. Local continuity of one of these upper bounds implies local continuity of $I(A: B)$ :

$$
\lim _{n \rightarrow \infty} H\left(\rho_{X}^{n}\right)=H\left(\rho_{X}^{0}\right)<+\infty \Rightarrow \lim _{n \rightarrow+\infty} I(A: B)_{\rho^{n}}=I(A: B)_{\rho^{0}}
$$

for any sequence $\left\{\rho_{A B}^{n}\right\}$ converging to a state $\rho_{A B}^{0}$, where $X$ is either $A$ or $B$ [29, Th.1].

A quantum channel $\Phi$ from a system $A$ to a system $B$ is a completely positive trace preserving linear map from $\mathfrak{T}\left(\mathcal{H}_{A}\right)$ into $\mathfrak{T}\left(\mathcal{H}_{B}\right)$ [15, 38. For any quantum channel $\Phi: A \rightarrow B$ the Stinespring theorem implies existence of a Hilbert space $\mathcal{H}_{E}$ and of an isometry $V_{\Phi}: \mathcal{H}_{A} \rightarrow \mathcal{H}_{B} \otimes \mathcal{H}_{E}$ such that

$$
\Phi(\rho)=\operatorname{Tr}_{E} V_{\Phi} \rho V_{\Phi}^{*}, \quad \rho \in \mathfrak{T}\left(\mathcal{H}_{A}\right) .
$$

The space $\mathcal{H}_{E}$ is called environment, its minimal dimension is called Choi rank of the channel $\Phi[15,38]$.

\footnotetext{
${ }^{2}$ The support $\operatorname{supp} \rho$ of a state $\rho$ is the closed subspace spanned by the eigenvectors of $\rho$ corresponding to its positive eigenvalues.
} 
Denote by $\mathfrak{F}(A, B)$ the set of all channels from $A$ to $B$ equipped with the topology of strong convergence defined by the family of seminorms $\Phi \mapsto\|\Phi(\rho)\|_{1}, \rho \in \mathfrak{S}\left(\mathcal{H}_{A}\right)$ [17. The strong convergence of a sequence $\left\{\Phi_{n}\right\}$ of channels in $\mathfrak{F}(A, B)$ to a channel $\Phi_{0} \in \mathfrak{F}(A, B)$ means that

$$
\lim _{n \rightarrow \infty} \Phi_{n}(\rho)=\Phi_{0}(\rho) \text { for all } \rho \in \mathfrak{S}\left(\mathcal{H}_{A}\right) .
$$

We will use the following important result.

Lemma 1. [7] If a sequence $\left\{\rho_{n}\right\}$ of states converges to a state $\rho_{0}$ w.r.t. the weak operator topology then the sequence $\left\{\rho_{n}\right\}$ of states converges to the state $\rho_{0}$ w.r.t. the trace norm.

We will also use the following simple

Lemma 2. If $h=f+g$, where $f$ and $g$ are lower semicontinuous lower bounded functions on a metric space $X$, then continuity of $h$ on a subset $X_{0} \subseteq X$ implies continuity of $f$ and $g$ on $X_{0}$.

\section{Extended QCMI and its properties}

\subsection{Tripartite system}

The quantum conditional mutual information (QCMI) of a state $\rho_{A B C}$ of a tripartite finite-dimensional system $A B C$ is defined as follows

$$
I(A: B \mid C)_{\rho} \doteq H\left(\rho_{A C}\right)+H\left(\rho_{B C}\right)-H\left(\rho_{A B C}\right)-H\left(\rho_{C}\right) .
$$

This quantity plays important role in different areas of quantum information theory [5, 6, 8, 11, 13, 32, 37, 35, 38, 42], it has the following basic properties:

1) $I(A: B \mid C)_{\rho} \geq 0$ for any state $\rho_{A B C}$ and $I(A: B \mid C)_{\rho}=0$ if and only if there is a channel $\Phi: C \rightarrow B C$ such that $\rho_{A B C}=\operatorname{Id}_{A} \otimes \Phi\left(\rho_{A C}\right)$ [13];

2) $I(A: B \mid C)_{\rho}=I(A C: B)_{\rho}-I(C: B)_{\rho}=I(A: B C)_{\rho}-I(A: C)_{\rho}$ for any state $\rho_{A B C}$;

3) monotonicity under local channels: $I(A: B \mid C)_{\rho} \geq I\left(A^{\prime}: B^{\prime} \mid C\right)_{\Phi_{A} \otimes \Phi_{B} \otimes \operatorname{Id} C}(\rho)$ for arbitrary quantum channels $\Phi_{A}: A \rightarrow A^{\prime}$ and $\Phi_{B}: B \rightarrow B^{\prime} \cdot 3$

4) additivity: $I\left(A A^{\prime}: B B^{\prime} \mid C C^{\prime}\right)_{\rho \otimes \rho^{\prime}}=I(A: B \mid C)_{\rho}+I\left(A^{\prime}: B^{\prime} \mid C^{\prime}\right)_{\rho^{\prime}}$ for any states $\rho_{A B C}$ and $\rho_{A^{\prime} B^{\prime} C^{\prime}}^{\prime}$;

5) duality: $I(A: B \mid C)_{\rho}=I(A: B \mid D)_{\rho}$ for any pure state $\rho_{A B C D}[8]$.

\footnotetext{
${ }^{3}$ The same inequality holds for any quantum operations $\Phi_{A}: A \rightarrow A^{\prime}$ and $\Phi_{B}: B \rightarrow B^{\prime}$ provided that the QCMI at any positive operator $\sigma$ is defined as $I(A: B \mid C)_{\sigma}=c I(A: B \mid C)_{\sigma / c}$, where $c=\operatorname{Tr} \sigma$. This can be derived from the monotonicity of relative entropy, f.i., by using formula (9.6) in [29].
} 
The nonnegativity of $I(A: B \mid C)_{\rho}$ is a basic result of quantum information theory well known as strong subadditivity of von Neumann entropy [21]. Devetak and Jard established an operational sense of QCMI as a cost of a quantum state redistribution protocol [8].

In infinite dimensions formula (9) may contain the uncertainty $+\infty-\infty$. In [29] it is shown that the r.h.s. of (9) has a unique lower semicontinuous extension to the space of all states of infinite-dimensional tripartite system possessing all basic properties of QCMI.

Theorem 1. 29] Let $A, B, C$ and $D$ be infinite-dimensional quantum systems. A) There exists a unique lower semicontinuous function $I_{\mathrm{e}}(A: B \mid C)_{\rho}$ on the set $\mathfrak{S}\left(\mathcal{H}_{A B C}\right)$ taking values in $[0,+\infty]$ such that:

- $I_{\mathrm{e}}(A: B \mid C)_{\rho}$ coincides with the r.h.s. of (9) if $H\left(\rho_{A B C}\right)$ and $H\left(\rho_{C}\right)$ are finite;

- the function $I_{\mathrm{e}}(A: B \mid C)_{\rho}$ possesses the above-stated properties 1-5 of QCMI

B) The function $I_{\mathrm{e}}(A: C \mid B)_{\rho}$ can be defined by one of the equivalent expression 5

$$
\begin{aligned}
& I_{\mathrm{e}}(A: B \mid C)_{\rho}=\sup _{P_{A}}\left[I(A: B C)_{Q \rho Q}-I(A: C)_{Q \rho Q}\right], \quad Q=P_{A} \otimes I_{B} \otimes I_{C}, \\
& I_{\mathrm{e}}(A: B \mid C)_{\rho}=\sup _{P_{B}}\left[I(A C: B)_{Q \rho Q}-I(B: C)_{Q \rho Q}\right], \quad Q=I_{A} \otimes P_{B} \otimes I_{C},
\end{aligned}
$$

where the suprema are over all finite rank projectors $P_{A} \in \mathfrak{B}\left(\mathcal{H}_{A}\right)$ and $P_{B} \in \mathfrak{B}\left(\mathcal{H}_{B}\right)$. C) For an arbitrary state $\rho_{A B C D}$ the following property is valid:

$$
I_{\mathrm{e}}(A: B \mid C)_{\rho}=\lim _{k \rightarrow+\infty} \lim _{l \rightarrow+\infty} I_{\mathrm{e}}(A: B \mid C)_{\rho^{k l t}}
$$

for $t=k$ and for $t=l$, where $\rho_{A B C D}^{k l t}$ is the state proportional to the operator

$$
P_{A}^{k} \otimes P_{B}^{k} \otimes P_{C}^{l} \otimes P_{D}^{t} \cdot \rho_{A B C D} \cdot P_{A}^{k} \otimes P_{B}^{k} \otimes P_{C}^{l} \otimes P_{D}^{t},
$$

$\left\{P_{A}^{k}\right\}_{k} \subset \mathfrak{B}\left(\mathcal{H}_{A}\right),\left\{P_{B}^{k}\right\}_{k} \subset \mathfrak{B}\left(\mathcal{H}_{B}\right),\left\{P_{C}^{l}\right\}_{l} \subset \mathfrak{B}\left(\mathcal{H}_{C}\right)$ and $\left\{P_{D}^{t}\right\}_{t} \subset \mathfrak{B}\left(\mathcal{H}_{D}\right)$ are sequences of projectors strongly converging to the unit operators $I_{A}, I_{B}, I_{C}$ and $I_{D}$ such that $\min \left\{\operatorname{rank} P_{A}^{k}, \operatorname{rank} P_{B}^{k}\right\}<+\infty$ for all $k$.

We will call the function $I_{\mathrm{e}}(A: B \mid C)_{\rho}$ the (extended) QCMI and will omit the subscript e. The benefits of using this function is pointed briefly in the Introduction.

The approximation property stated in part $\mathrm{C}$ of Theorem 1 allows to prove for the extended QCMI the relations valid for the QCMI in the finite-dimensional case. For example, it allows to show that the following relation

$$
I(A D: B E \mid C)_{\rho}=I(A D: E \mid B C)_{\rho}+I(D: B \mid A C)_{\rho}+I(A: B \mid C)_{\rho}
$$

\footnotetext{
${ }^{4}$ The first and the second equalities in property 2 are valid if $I(B: C)<+\infty$ and $I(A: C)<+\infty$ correspondingly.

${ }^{5} \mathrm{It}$ is assumed that $I(X: Y)_{Q \rho Q}=[\operatorname{Tr} Q \rho] I(X: Y)_{\frac{Q \rho Q}{\operatorname{Tr} Q \rho}}$.
} 
holds for any state $\rho_{A B C D E}$ (with possible values $+\infty$ in both sides).

A similar way is used to prove that

$$
\left|p I(A: B \mid C)_{\rho}+(1-p) I(A: B \mid C)_{\sigma}-I(A: B \mid C)_{p \rho+(1-p) \sigma}\right| \leq h_{2}(p)
$$

for any $p \in(0,1)$ and any states $\rho_{A B C}, \sigma_{A B C}$ with finite $I(A: B \mid C)_{\rho}, I(A: B \mid C)_{\sigma}$, where $h_{2}(p)$ is the binary entropy [31.

The lower semicontinuity of $I(A: B \mid C)$ on $\mathfrak{S}\left(\mathcal{H}_{A B C}\right)$ means that

$$
\liminf _{n \rightarrow+\infty} I(A: B \mid C)_{\rho^{n}} \geq I(A: B \mid C)_{\rho^{0}}
$$

for any sequence $\left\{\rho_{A B C}^{n}\right\}$ converging to a state $\rho_{A B C}^{0}$. Physically, this limit relation shows that the conditional total correlation can not be increased by passing to a limit. It implies that the set of all states $\rho_{A B C}$ determined by the inequality

$$
I(A: B \mid C)_{\rho} \leq \epsilon
$$

is closed for any $\epsilon \geq 0$. The existence of the Fawzi-Renner recovery channel [11], i.e. a channel $\Phi: C \rightarrow B C$ such that

$$
F\left(\rho_{A B C}, \operatorname{Id}_{A} \otimes \Phi\left(\rho_{A C}\right)\right) \geq 2^{-\epsilon / 2}
$$

where $F(\varrho, \varsigma) \doteq\|\sqrt{\varrho} \sqrt{\varsigma}\|_{1}$ is the quantum fidelity for states $\varrho$ and $\varsigma$, is proved in [29] for arbitrary state $\rho_{A B C}$ satisfying (15) by using the approximation technique based on the compactness criterion for families of channels in the strong convergence topology 6

If either $I(A: C)_{\rho}$ or $I(B: C)_{\rho}$ is finite then expressions (10) and (11) are reduced, respectively, to the following well known formulae

$$
\begin{aligned}
& I(A: B \mid C)_{\rho}=I(A: B C)_{\rho}-I(A: C)_{\rho}, \\
& I(A: B \mid C)_{\rho}=I(A C: B)_{\rho}-I(B: C)_{\rho} .
\end{aligned}
$$

These formulae and upper bound (7) imply that

$$
I(A: B \mid C)_{\rho} \leq 2 \min \left\{H\left(\rho_{A}\right), H\left(\rho_{B}\right), H\left(\rho_{A C}\right), H\left(\rho_{B C}\right)\right\} .
$$

By formulae (17) and (18) the lower semicontinuity of QCMI, Lemma 2 and continuity condition (8) for the quantum mutual information imply the following continuity conditions for QCMI.

Corollary 1. [29] If $\left\{\rho_{A B C}^{n}\right\}$ is a sequence converging to a state $\rho_{A B C}^{0}$ such that $\lim _{n \rightarrow+\infty} H\left(\rho_{X}^{n}\right)=H\left(\rho_{X}^{0}\right)<+\infty$, where $X$ is one of the systems $A, B, A C$ and $B C$ then

$$
\lim _{n \rightarrow+\infty} I(A: B \mid C)_{\rho^{n}}=I(A: B \mid C)_{\rho^{0}}<+\infty .
$$

\footnotetext{
${ }^{6}$ In [11, 34] the existence of such a channel was proved only for states with finite marginal entropies.
} 
These conditions will be substantially strengthened in Section 5.1.1 (Corollary 3 ).

By Theorem 1 the functions $\rho \mapsto I(A D: E \mid B C)_{\rho}$ and $\rho \mapsto I(D: B \mid A C)_{\rho}$ are lower semicontinuous on the set of all states in $\mathfrak{S}\left(\mathcal{H}_{A B C D E}\right)$, where $A, B, C, D$ and $E$ are arbitrary infinite-dimensional systems. Thus, the relation (13) implies the following

Corollary 2. The function $\rho \mapsto\left[I(A D: B E \mid C)_{\rho}-I(A: B \mid C)_{\rho}\right]$ is lower semicontinuous on the set $\left\{\rho \in \mathfrak{S}\left(\mathcal{H}_{A B C D E}\right) \mid I(A: B \mid C)_{\rho}<+\infty\right\}$.

This corollary will be essentially used in Section 4 .

\subsection{Multipartite system}

The quantum conditional mutual information of a state $\rho_{A_{1} \ldots A_{m} C}$ of a finite-dimensional multipartite system $A_{1} \ldots A_{m} C$ is defined as follows (cf. [23, 14, 39, 11, 41])

$$
\begin{aligned}
I\left(A_{1}: \ldots: A_{m} \mid C\right)_{\rho} & \doteq \sum_{k=1}^{m} H\left(A_{k} \mid C\right)_{\rho}-H\left(A_{1} \ldots A_{m} \mid C\right)_{\rho} \\
& =\sum_{k=1}^{m-1} H\left(A_{k} \mid C\right)_{\rho}-H\left(A_{1} \ldots A_{m-1} \mid A_{m} C\right)_{\rho} .
\end{aligned}
$$

Its nonnegativity and other basic properties can be derived from the corresponding properties of the tripartite QCMI by using the representation (cf. [41])

$$
\begin{aligned}
I\left(A_{1}: \ldots: A_{m} \mid C\right)_{\rho}=I\left(A_{2}: A_{1} \mid C\right)_{\rho} & +I\left(A_{3}: A_{1} A_{2} \mid C\right)_{\rho}+\ldots \\
& +I\left(A_{m}: A_{1} \ldots A_{m-1} \mid C\right)_{\rho} .
\end{aligned}
$$

By using representation (21) and the extended QCMI described in Theorem 1 one can define QCMI for any state of an infinite-dimensional system $A_{1} \ldots A_{m} C$.

Proposition 1. [29] Let $A_{1}, \ldots A_{m}$ and $C$ be infinite-dimensional quantum systems. A) There is a unique lower semicontinuous function $I_{\mathrm{e}}\left(A_{1}: \ldots: A_{m} \mid C\right)_{\rho}$ on the set $\mathfrak{S}\left(\mathcal{H}_{A_{1} \ldots A_{m} C}\right)$ coinciding with the r.h.s. of (20) for any state $\rho_{A_{1} \ldots A_{m} C}$ with finite marginal entropies and possessing the analogs of above-stated properties 1-4 of QCMI. This function can be defined by formula (21) in which each summand $I(X: Y \mid C)_{\rho}$ coincides with the function $I_{\mathrm{e}}(X: Y \mid C)_{\rho}$ described in Theorem 1 .

B) For an arbitrary state $\rho_{A_{1} \ldots A_{m} C D}$ the following property is valid:

$$
I_{\mathrm{e}}\left(A_{1}: \ldots: A_{m} \mid C\right)_{\rho}=\lim _{k \rightarrow+\infty} \lim _{l \rightarrow+\infty} I_{\mathrm{e}}\left(A_{1}: \ldots: A_{m} \mid C\right)_{\rho^{k l t}}
$$

for $t=k$ and for $t=l$, where $\rho_{A_{1} \ldots A_{m} C D}^{k l t}$ is the state proportional to the operator

$$
P_{A_{1}}^{k} \otimes \ldots \otimes P_{A_{m}}^{k} \otimes P_{C}^{l} \otimes P_{D}^{t} \cdot \rho_{A_{1} \ldots A_{m} C D} \cdot P_{A_{1}}^{k} \otimes \ldots \otimes P_{A_{m}}^{k} \otimes P_{C}^{l} \otimes P_{D}^{t},
$$

$\left\{P_{A_{i}}^{k}\right\}_{k} \subset \mathfrak{B}\left(\mathcal{H}_{A_{i}}\right), i=\overline{1, m},\left\{P_{C}^{l}\right\}_{l} \subset \mathfrak{B}\left(\mathcal{H}_{C}\right)$ and $\left\{P_{D}^{t}\right\}_{t} \subset \mathfrak{B}\left(\mathcal{H}_{D}\right)$ are sequences of projectors strongly converging to the unit operators $I_{A_{i}}, i=\overline{1, m}, I_{C}$ and $I_{D}$ such that $\min _{1 \leq j \leq m} \sum_{i \neq j} \operatorname{rank} P_{A_{i}}^{k}<+\infty$ for all $k$. 
The approximation property stated in part B of Proposition 1 allows to prove for the function $I_{\mathrm{e}}\left(A_{1}: \ldots: A_{m} \mid C\right)$ the relations valid for the multipartite QCMI in the finite-dimensional case. It allows to prove, in particular, that representation (21) is valid for $I_{\mathrm{e}}\left(A_{1}: \ldots: A_{m} \mid C\right)_{\rho}$ with arbitrarily permuted indexes $1, \ldots, m$ in the right hand side (provided each summand $I(X: Y \mid Z)_{\rho}$ coincides with $\left.I_{\mathrm{e}}(X: Y \mid Z)_{\rho}\right)$ [29].

We will call the function $I_{\mathrm{e}}\left(A_{1}: \ldots: A_{m} \mid C\right)$ the (extended) multipartite QCMI and will omit the subscript e.

We will use the m-partite version of inequality (14): let $\rho_{A_{1} \ldots A_{m} C}$ and $\sigma_{A_{1} \ldots A_{m} C}$ be states such that $R=I\left(A_{1}: \ldots: A_{m} \mid C\right)_{\rho}$ and $S=I\left(A_{1}: \ldots: A_{m} \mid C\right)_{\sigma}$ are finite. Then

$$
-h_{2}(p) \leq I\left(A_{1}: \ldots: A_{m} \mid C\right)_{p \rho+(1-p) \sigma}-[p R+(1-p) S] \leq(m-1) h_{2}(p)
$$

for any $p \in(0,1)$, where $h_{2}(p)$ is the binary entropy.

If $\rho_{A_{1} \ldots A_{m} C}$ and $\sigma_{A_{1} \ldots A_{m} C}$ are states with finite marginal entropies then inequality (23) can be proved by using the second expression in (20), concavity of the conditional entropy and inequality (3). The validity of (23) for arbitrary states $\rho_{A_{1} \ldots A_{m} C}$ and

$\sigma_{A_{1} \ldots A_{m} C}$ with finite QCMI can be shown by using the approximation property stated in part B of Proposition 1 .

\section{Lower semicontinuity of the loss of QCMI under local channels}

\subsection{Tripartite system}

One of the basic properties of QCMI is the monotonicity under local channels. It means that

$$
I(A: B \mid C)_{\rho} \geq I\left(A^{\prime}: B^{\prime} \mid C\right)_{\Phi_{A} \otimes \Phi_{B} \otimes \operatorname{Id}_{C}(\rho)}
$$

for any state $\rho_{A B C}$ and arbitrary channels $\Phi_{A}: A \rightarrow A^{\prime}$ and $\Phi_{B}: B \rightarrow B^{\prime}$. So, the quantity

$$
\Delta\left(\rho_{A B C}, \Phi_{A}, \Phi_{B}\right) \doteq I(A: B \mid C)_{\rho}-I\left(A^{\prime}: B^{\prime} \mid C\right)_{\Phi_{A} \otimes \Phi_{B} \otimes \operatorname{Id}_{C}(\rho)}
$$

is well defined as a nonnegative number or $+\infty$ for any $\rho_{A B C}, \Phi_{A}$ and $\Phi_{B}$ such that $I\left(A^{\prime}: B^{\prime} \mid C\right)_{\Phi_{A} \otimes \Phi_{B} \otimes \operatorname{Id}_{C}(\rho)}<+\infty$. We will call it the loss of QCMI under local channels.

To analyse the action of a local channel on the conditioning system we will consider the quantity

$$
\Delta^{\mathrm{c}}\left(\rho_{A B C}, \Phi_{C}\right) \doteq I(A: B \mid C)_{\rho}-I\left(A: B \mid C^{\prime}\right)_{\operatorname{Id}_{A B} \otimes \Phi_{C}(\rho)}
$$

which can take values of different sign (in contrast to $\Delta\left(\rho_{A B C}, \Phi_{A}, \Phi_{B}\right)$ ).

We will consider $\Delta\left(\rho_{A B C}, \Phi_{A}, \Phi_{B}\right)$ as a function of a triplet $\left(\rho_{A B C}, \Phi_{A}, \Phi_{B}\right)$ and $\Delta^{\mathrm{c}}\left(\rho_{A B C}, \Phi_{C}\right)$ as a function of a pair $\left(\rho_{A B C}, \Phi_{C}\right)$. 
Theorem 2. A) The function $\Delta\left(\rho_{A B C}, \Phi_{A}, \Phi_{B}\right)$ is lower semicontinuous on the set 7 $\left\{\left(\rho_{A B C}, \Phi_{A}, \Phi_{B}\right) \in \mathfrak{S}\left(\mathcal{H}_{A B C}\right) \times \mathfrak{F}\left(A, A^{\prime}\right) \times \mathfrak{F}\left(B, B^{\prime}\right) \mid I\left(A^{\prime}: B^{\prime} \mid C\right)_{\Phi_{A} \otimes \Phi_{B} \otimes \operatorname{Id}_{C}(\rho)}<+\infty\right\}$. B) The function $\Delta^{\mathrm{c}}\left(\rho_{A B C}, \Phi_{C}\right)$ is lower semicontinuous and finite on the set

$$
\left\{\left(\rho_{A B C}, \Phi_{C}\right) \in \mathfrak{S}_{\mathrm{c}} \times \mathfrak{F}\left(C, C^{\prime}\right) \mid I(A: B \mid C)_{\rho}<+\infty\right\}
$$

where $\mathfrak{S}_{\mathrm{c}}$ is any subset of $\mathfrak{S}\left(\mathcal{H}_{A B C}\right)$ on which the function $\rho_{A B C} \mapsto H\left(\rho_{C}\right)$ is continuous. This function is bounded from below by the value $-2 H\left(\rho_{C}\right)$.

C) The function $\Delta^{\mathrm{c}}\left(\rho_{A B C}, \Phi_{C}\right)$ is continuous and bounded on the set

$$
\left\{\left(\rho_{A B C}, \Phi_{C}\right) \in \mathfrak{S}\left(\mathcal{H}_{A B C}\right) \times \mathfrak{F}_{k}\left(C, C^{\prime}\right) \mid I(A: B \mid C)_{\rho}<+\infty\right\}
$$

for each $k \in \mathbb{N}$, where $\mathfrak{F}_{k}\left(C, C^{\prime}\right)$ is the subsets of $\mathfrak{F}\left(C, C^{\prime}\right)$ consisting of channels with Choi rank not exceeding $k$. The modulus of $\Delta^{\mathrm{c}}\left(\rho_{A B C}, \Phi_{C}\right)$ on this set does not exceed $2 \log k$.

Theorem 2A states that

$$
\liminf _{n \rightarrow+\infty} \Delta\left(\rho_{A B C}^{n}, \Phi_{A}^{n}, \Phi_{B}^{n}\right) \geq \Delta\left(\rho_{A B C}^{0}, \Phi_{A}^{0}, \Phi_{B}^{0}\right)
$$

for any sequence $\left\{\rho_{A B C}^{n}\right\}$ converging to a state $\rho_{A B C}^{0}$ and any sequences $\left\{\Phi_{A}^{n}\right\} \subset \mathfrak{F}\left(A, A^{\prime}\right)$ and $\left\{\Phi_{B}^{n}\right\} \subset \mathfrak{F}\left(B, B^{\prime}\right)$ strongly converging, respectively, to channels $\Phi_{A}^{0}$ and $\Phi_{B}^{0}$ provided that $I\left(A^{\prime}: B^{\prime} \mid C\right)_{\Phi_{A}^{n} \otimes \Phi_{B}^{n} \otimes \operatorname{Id}_{C}\left(\rho^{n}\right)}<+\infty$ for all $n \geq 0$ (otherwise the quantity $\Delta\left(\rho_{A B C}^{n}, \Phi_{A}^{n}, \Phi_{B}^{n}\right)$ is not defined $)$.

Theorem $2 \mathrm{~B}$ states that

$$
\liminf _{n \rightarrow+\infty} \Delta^{\mathrm{c}}\left(\rho_{A B C}^{n}, \Phi_{C}^{n}\right) \geq \Delta^{\mathrm{c}}\left(\rho_{A B C}^{0}, \Phi_{C}^{0}\right) \geq-2 H\left(\rho_{C}^{0}\right)
$$

for any sequence $\left\{\rho_{A B C}^{n}\right\}$ converging to a state $\rho_{A B C}^{0}$ and any sequence $\left\{\Phi_{C}^{n}\right\} \subset \mathfrak{F}\left(C, C^{\prime}\right)$ strongly converging to a channel $\Phi_{C}^{0}$ provided that the sequence $\left\{H\left(\rho_{C}^{n}\right)\right\}$ tends to $H\left(\rho_{C}^{0}\right)<+\infty$ and $I(A: B \mid C)_{\rho^{n}}<+\infty$ for all $n \geq 0$.

Theorem $2 \mathrm{C}$ states that

$$
\lim _{n \rightarrow+\infty} \Delta^{\mathrm{c}}\left(\rho_{A B C}^{n}, \Phi_{C}^{n}\right)=\Delta^{\mathrm{c}}\left(\rho_{A B C}^{0}, \Phi_{C}^{0}\right) \quad \text { and } \quad\left|\Delta^{\mathrm{c}}\left(\rho_{A B C}^{0}, \Phi_{C}^{0}\right)\right| \leq 2 \log k
$$

for any sequence $\left\{\rho_{A B C}^{n}\right\}$ converging to a state $\rho_{A B C}^{0}$ and any sequence $\left\{\Phi_{C}^{n}\right\} \subset \mathfrak{F}\left(C, C^{\prime}\right)$ strongly converging to a channel $\Phi_{C}^{0}$ provided that the Choi rank of all the channels $\Phi_{C}^{n}$ does not exceed $k$ and $I(A: B \mid C)_{\rho^{n}}<+\infty$ for all $n \geq 0$.

Proof. A) Let $\left\{\rho_{A B C}^{n}\right\}$ be a sequence of states converging to a state $\rho_{A B C}^{0}$. Let $\left\{\Phi_{A}^{n}\right\}$ and $\left\{\Phi_{B}^{n}\right\}$ be sequences of channels in $\mathfrak{F}\left(A, A^{\prime}\right)$ and in $\mathfrak{F}\left(B, B^{\prime}\right)$ strongly converging to

\footnotetext{
${ }^{7} \mathfrak{F}\left(X, X^{\prime}\right)$ is the set of all quantum channels from a system $X$ to a system $X^{\prime}$ equipped with the strong convergence topology (see Section 2).
} 
channels $\Phi_{A}^{0}$ and $\Phi_{B}^{0}$ correspondingly such that $I\left(A^{\prime}: B^{\prime} \mid C\right)_{\Phi_{A}^{n} \otimes \Phi_{B}^{n} \otimes \operatorname{Id}_{C}\left(\rho^{n}\right)}<+\infty$ for all $n \geq 0$. By Theorem $2 \mathrm{~B}$ in [33] there exist separable Hilbert spaces $\mathcal{H}_{D}$ and $\mathcal{H}_{E}$ and sequences of isometries $V_{n}: \mathcal{H}_{A} \rightarrow \mathcal{H}_{A^{\prime} D}$ and $U_{n}: \mathcal{H}_{B} \rightarrow \mathcal{H}_{B^{\prime} E}$ strongly converging to isometries $V_{0}$ and $U_{0}$ such that

$$
\Phi_{A}^{n}(\varrho)=\operatorname{Tr}_{D} V_{n} \varrho V_{n}^{*} \quad \text { and } \quad \Phi_{B}^{n}(\varsigma)=\operatorname{Tr}_{E} U_{n} \varsigma U_{n}^{*}
$$

for any $\varrho \in \mathfrak{S}\left(\mathcal{H}_{A}\right), \varsigma \in \mathfrak{S}\left(\mathcal{H}_{B}\right)$ and all $n \geq 0$.

The sequence of states $\sigma_{A^{\prime} B^{\prime} C D E}^{n}=V_{n} \otimes U_{n} \otimes I_{C} \cdot \rho_{A B C}^{n} \cdot V_{n}^{*} \otimes U_{n}^{*} \otimes I_{C}$ converges to the state $\sigma_{A^{\prime} B^{\prime} C D E}^{0}=V_{0} \otimes U_{0} \otimes I_{C} \cdot \rho_{A B C}^{0} \cdot V_{0}^{*} \otimes U_{0}^{*} \otimes I_{C}$. Since

$$
I(A: B \mid C)_{\rho^{n}}=I\left(A^{\prime} D: B^{\prime} E \mid C\right)_{\sigma^{n}} \quad \text { and } \quad I\left(A^{\prime}: B^{\prime} \mid C\right)_{\Phi_{A}^{n} \otimes \Phi_{B}^{n} \otimes \operatorname{Id}_{C}\left(\rho^{n}\right)}=I\left(A^{\prime}: B^{\prime} \mid C\right)_{\sigma^{n}}
$$

for all $n \geq 0$, relation (24) follows from Corollary 2 in Section 3 .

B) Let $\left\{\rho_{A B C}^{n}\right\}$ be a sequence of states converging to a state $\rho_{A B C}^{0}$ such that $I(A: B \mid C)_{\rho^{n}}<+\infty$ for all $n \geq 0$ and $\left\{\Phi_{C}^{n}\right\}$ a sequences of channels in $\mathfrak{F}\left(C, C^{\prime}\right)$ strongly converging to a channel $\Phi_{C}^{0}$. We have to prove (25) assuming that the sequence $\left\{H\left(\rho_{C}^{n}\right)\right\}$ tends to $H\left(\rho_{C}^{0}\right)<+\infty$.

By Theorem 2B in [33] there is a separable Hilbert space $\mathcal{H}_{E}$ and a sequence of isometries $V_{n}: \mathcal{H}_{C} \rightarrow \mathcal{H}_{C^{\prime} E}$ strongly converging to an isometry $V_{0}$ such that

$$
\Phi_{C}^{n}(\varrho)=\operatorname{Tr}_{E} V_{n} \varrho V_{n}^{*} \quad \text { for all } \varrho \in \mathfrak{S}\left(\mathcal{H}_{C}\right) \text { and } n \geq 0
$$

The sequence of states $\sigma_{A B C^{\prime} E}^{n}=I_{A B} \otimes V_{n} \cdot \rho_{A B C}^{n} \cdot I_{A B} \otimes V_{n}^{*}$ converges to a state $\sigma_{A B C^{\prime} E}^{0}=I_{A B} \otimes V_{0} \cdot \rho_{A B C}^{0} \cdot I_{A B} \otimes V_{0}^{*}$. Since

$$
I(A: B \mid C)_{\rho^{n}}=I\left(A: B \mid C^{\prime} E\right)_{\sigma^{n}} \quad \text { and } \quad I\left(A: B \mid C^{\prime}\right)_{\operatorname{Id}_{A B} \otimes \Phi_{C}^{n}\left(\rho^{n}\right)}=I\left(A: B \mid C^{\prime}\right)_{\sigma^{n}},
$$

Lemma 3 below implies that

$$
\Delta^{\mathrm{c}}\left(\rho_{A B C}^{n}, \Phi_{C}^{n}\right)=I\left(B: E \mid A C^{\prime}\right)_{\sigma^{n}}-I\left(B: E \mid C^{\prime}\right)_{\sigma^{n}}
$$

for all $n \geq 0$ provided that the last term is finite.

By the assumption we have $\lim _{n \rightarrow+\infty} H\left(\sigma_{C^{\prime} E}^{n}\right)=H\left(\sigma_{C^{\prime} E}^{0}\right)<+\infty$, since $H\left(\sigma_{C^{\prime} E}^{n}\right)=$ $H\left(V_{n} \rho_{C}^{n} V_{n}^{*}\right)=H\left(\rho_{C}^{n}\right)$ for all $n \geq 0$. Hence Corollary 1 and upper bound (19) imply that

$$
\lim _{n \rightarrow+\infty} I\left(B: E \mid C^{\prime}\right)_{\sigma^{n}}=I\left(B: E \mid C^{\prime}\right)_{\sigma^{0}} \leq 2 H\left(\sigma_{E C^{\prime}}^{0}\right)=2 H\left(\rho_{C}^{0}\right) .
$$

Thus, relation (25) follows from (27) and the lower semicontinuity of QCMI.

C) By Theorem 2B in [33] in this case we can repeat the arguments from the proof of part $\mathrm{B}$ with $k$-dimensional system $E$. Hence the relations

$$
\lim _{n \rightarrow+\infty} I\left(B: E \mid A C^{\prime}\right)_{\sigma^{n}}=I\left(B: E \mid A C^{\prime}\right)_{\sigma^{0}}<+\infty
$$

and (28) hold by Corollary 1. These relations and (27) imply (26). 
The upper bound on $\left|\Delta^{\mathrm{c}}\left(\rho_{A B C}, \Phi_{C}\right)\right|$ follows from the expression similar to (27), since upper bound (19) implies that

$$
I\left(B: E \mid A C^{\prime}\right)_{\sigma}, I\left(B: E \mid C^{\prime}\right)_{\sigma} \leq 2 \log \operatorname{dim} \mathcal{H}_{E}
$$

for any state $\sigma$ in $\mathfrak{S}\left(\mathcal{H}_{A B C^{\prime} E}\right)$.

Lemma 3. Let $A, B, C$ and $D$ be arbitrary quantum systems. Then the equality

$$
I(A: B \mid C D)_{\rho}-I(A: B \mid C)_{\rho}=I(D: B \mid A C)_{\rho}-I(D: B \mid C)_{\rho}
$$

holds for any state $\rho$ in $\mathfrak{S}\left(\mathcal{H}_{A B C D}\right)$ provided that both sides of (29) are well defined (do not contain the uncertainty $+\infty-\infty)$.

Proof. Equality (29) is easily verified for any state $\rho$ with finite marginal entropies (by using formula (9) for QCMI). Its validity for arbitrary state $\rho$ can be shown by using two step approximation.

Let $\rho$ be a state such that $H\left(\rho_{B}\right)<+\infty$. Let $\left\{\Phi_{X}^{n}\right\}$ be sequences of channels strongly converging to the identity channels $\operatorname{Id}_{X}, X=A, C, D$, such that any channel $\Phi_{X}^{n}$ has a finite-dimensional output. The sequence of states $\rho^{n}=\Phi_{A}^{n} \otimes \operatorname{Id}_{B} \otimes \Phi_{C}^{n} \otimes \Phi_{D}^{n}(\rho)$ having finite marginal entropies tends to the state $\rho$. Corollary 1 implies that

$$
\begin{aligned}
& I(A: B \mid C D)_{\rho^{n}} \rightarrow I(A: B \mid C D)_{\rho}, \quad I(A: B \mid C)_{\rho^{n}} \rightarrow I(A: B \mid C)_{\rho} \\
& I(D: B \mid A C)_{\rho^{n}} \rightarrow I(D: B \mid A C)_{\rho}, \quad I(D: B \mid C)_{\rho^{n}} \rightarrow I(D: B \mid C)_{\rho}
\end{aligned}
$$

as $n \rightarrow+\infty$, where all the limits are finite. So, since equality (29) holds for the state $\rho^{n}$ for each $n$, it holds for the state $\rho$.

Now the validity of equality (29) for arbitrary state $\rho$ at which both parts of (29) are well defined can be easily shown by using the approximating property from part $\mathrm{C}$ of Theorem 1, $\square$.

\subsection{Multipartite system}

The monotonicity of the extended multipartite QCMI under local channels means that

$$
I\left(A_{1}: \ldots: A_{m} \mid C\right)_{\rho} \geq I\left(A_{1}^{\prime}: \ldots: A_{m}^{\prime} \mid C\right)_{\Phi_{A_{1}} \otimes \ldots \otimes \Phi_{A_{m}} \otimes \mathrm{Id}_{C}(\rho)}
$$

for any state $\rho_{A_{1} \ldots A_{m} C}$ and arbitrary channels $\Phi_{A_{k}}: A_{k} \rightarrow A_{k}^{\prime}, k=\overline{1, m}$. So, the quantity

$$
\Delta\left(\rho_{A_{1} \ldots A_{m} C}, \Phi_{A_{1}}, \ldots, \Phi_{A_{m}}\right) \doteq I\left(A_{1}: \ldots: A_{m} \mid C\right)_{\rho}-I\left(A_{1}^{\prime}: \ldots: A_{m}^{\prime} \mid C\right)_{\Phi_{A_{1}} \otimes \ldots \otimes \Phi_{A_{m}} \otimes \operatorname{Id}_{C}(\rho)}
$$

is well defined as a nonnegative number or $+\infty$ for any $\Phi_{A_{1}, \ldots, \Phi_{A_{m}}}$ and $\rho_{A_{1} \ldots A_{m} C}$ such that $I\left(A_{1}^{\prime}: \ldots: A_{m}^{\prime} \mid C\right)_{\Phi_{A_{1}} \otimes \ldots \otimes \Phi_{A_{m}} \otimes \operatorname{Id}_{C}(\rho)}<+\infty$. We will call it the loss of the multipartite QCMI under local channels. 
To analyse the action of a local channel on the conditioning system we will consider the quantity

$$
\Delta^{\mathrm{c}}\left(\rho_{A_{1} \ldots A_{m} C}, \Phi_{C}\right) \doteq I\left(A_{1}: \ldots: A_{m} \mid C\right)_{\rho}-I\left(A_{1}: \ldots: A_{m} \mid C^{\prime}\right)_{\operatorname{Id}_{A_{1} \ldots A_{m}} \otimes \Phi_{C}(\rho)}
$$

which can take values of different sign (in contrast to $\Delta\left(\rho_{A_{1} \ldots A_{m} C}, \Phi_{A_{1}}, \ldots, \Phi_{A_{m}}\right)$ ).

Denote by $\mathfrak{F}_{l}\left(\left\{A_{k}\right\},\left\{A_{k}^{\prime}\right\}\right)$ the Cartesian product of the spaces $\mathfrak{F}\left(A_{1}, A_{1}^{\prime}\right), \ldots, \mathfrak{F}\left(A_{m}, A_{m}^{\prime}\right)$ of all local channels equipped with the strong convergence topology (see Section 2).

Theorem 3. A) The function $\Delta\left(\rho_{A_{1} \ldots A_{m} C}, \Phi_{A_{1}}, \ldots, \Phi_{A_{m}}\right)$ is lower semicontinuous on the set

$\left\{\left(\rho_{A_{1} \ldots A_{m} C}, \Phi_{A_{1}}, \ldots, \Phi_{A_{m}}\right) \in \mathfrak{S}\left(\mathcal{H}_{A_{1} \ldots A_{m} C}\right) \times \mathfrak{F}_{l}\left(\left\{A_{k}\right\},\left\{A_{k}^{\prime}\right\}\right) \mid I\left(A_{1}^{\prime}: \ldots: A_{m}^{\prime} \mid C\right)_{\rho^{\prime}}<+\infty\right\}$ where $\rho_{A_{1}^{\prime} \ldots A_{m}^{\prime} C}^{\prime}=\Phi_{A_{1}} \otimes \ldots \otimes \Phi_{A_{m}} \otimes \operatorname{Id}_{C}\left(\rho_{A_{1} \ldots A_{m} C}\right)$.

B) The function $\Delta^{\mathrm{c}}\left(\rho_{A_{1} \ldots A_{m} C}, \Phi_{C}\right)$ is lower semicontinuous and finite on the set

$$
\left\{\left(\rho_{A_{1} \ldots A_{m} C}, \Phi_{C}\right) \in \mathfrak{S}_{\mathrm{c}} \times \mathfrak{F}\left(C, C^{\prime}\right) \mid I\left(A_{1}: \ldots: A_{m} \mid C\right)_{\rho}<+\infty\right\},
$$

where $\mathfrak{S}_{\mathrm{c}}$ is any subset of $\mathfrak{S}\left(\mathcal{H}_{A_{1} \ldots A_{m} C}\right)$ on which the function $\rho_{A_{1} \ldots A_{m} C} \mapsto H\left(\rho_{C}\right)$ is continuous. This function is bounded from below by the value $-2(m-1) H\left(\rho_{C}\right)$.

C) The function $\Delta^{\mathrm{c}}\left(\rho_{A_{1} \ldots A_{m} C}, \Phi_{C}\right)$ is continuous and bounded on the set

$$
\left\{\left(\rho_{A_{1} \ldots A_{m} C}, \Phi_{C}\right) \in \mathfrak{S}\left(\mathcal{H}_{A_{1} \ldots A_{m} C}\right) \times \mathfrak{F}_{k}\left(C, C^{\prime}\right) \mid I\left(A_{1}: \ldots: A_{m} \mid C\right)_{\rho}<+\infty\right\}
$$

for each $k \in \mathbb{N}$, where $\mathfrak{F}_{k}\left(C, C^{\prime}\right)$ is the subset of $\mathfrak{F}\left(C, C^{\prime}\right)$ consisting of channels with the Choi rank not exceeding $k$. The modulus of $\Delta^{\mathrm{c}}\left(\rho_{A_{1} \ldots A_{m} C}, \Phi_{C}\right)$ on this set does not exceed $2(m-1) \log k$.

Proof. All the parts of Theorem 3 can be easily derived from the corresponding parts of Theorem 2 by using representation (21) $\square$.

Theorem 3 A states that

$$
\liminf _{n \rightarrow+\infty} \Delta\left(\rho_{A_{1} \ldots A_{m} C}^{n}, \Phi_{A_{1}}^{n}, \ldots, \Phi_{A_{m}}^{n}\right) \geq \Delta\left(\rho_{A_{1} \ldots A_{m} C}^{0}, \Phi_{A_{1}}^{0}, \ldots, \Phi_{A_{m}}^{0}\right)
$$

for arbitrary sequence $\left\{\rho_{A_{1} \ldots A_{m} C}^{n}\right\}$ converging to a state $\rho_{A_{1} \ldots A_{m} C}^{0}$ and any sequences $\left\{\Phi_{A_{1}}^{n}\right\} \subset \mathfrak{F}\left(A_{1}, A_{1}^{\prime}\right), \ldots,\left\{\Phi_{A_{m}}^{n}\right\} \subset \mathfrak{F}\left(A_{m}, A_{m}^{\prime}\right)$ strongly converging, respectively, to channels $\Phi_{A_{1}}^{0}, \ldots, \Phi_{A_{m}}^{0}$ provided that $I\left(A_{1}^{\prime}: \ldots: A_{m}^{\prime} \mid C\right)_{\Phi_{A_{1}} \otimes \ldots \otimes \Phi_{A_{m}} \otimes \operatorname{Id}_{C}\left(\rho^{n}\right)}<+\infty$ for all $n \geq 0$.

Theorem $3 \mathrm{~B}$ states that

$$
\liminf _{n \rightarrow+\infty} \Delta^{\mathrm{c}}\left(\rho_{A_{1} \ldots A_{m} C}^{n}, \Phi_{C}^{n}\right) \geq \Delta^{\mathrm{c}}\left(\rho_{A_{1} \ldots A_{m} C}^{0}, \Phi_{C}^{0}\right) \geq-2(m-1) H\left(\rho_{C}^{0}\right)
$$

for arbitrary sequence $\left\{\rho_{A_{1} \ldots A_{m} C}^{n}\right\}$ converging to a state $\rho_{A_{1} \ldots A_{m} C}^{0}$ and any sequence $\left\{\Phi_{c}^{n}\right\} \subset \mathfrak{F}\left(C, C^{\prime}\right)$ strongly converging to a channel $\Phi_{C}^{0}$ provided that the sequence $\left\{H\left(\rho_{C}^{n}\right)\right\}$ tends to $H\left(\rho_{C}^{0}\right)<+\infty$ and $I\left(A_{1}: \ldots: A_{m} \mid C\right)_{\rho^{n}}<+\infty$ for all $n \geq 0$. 
Theorem $3 \mathrm{C}$ states that

$\lim _{n \rightarrow+\infty} \Delta^{\mathrm{c}}\left(\rho_{A_{1} \ldots A_{m} C}^{n}, \Phi_{C}^{n}\right)=\Delta^{\mathrm{c}}\left(\rho_{A_{1} \ldots A_{m} C}^{0}, \Phi_{C}^{0}\right) \quad$ and $\quad\left|\Delta^{\mathrm{c}}\left(\rho_{A_{1} \ldots A_{m} C}^{0}, \Phi_{C}^{0}\right)\right| \leq 2(m-1) \log k$

for arbitrary sequence $\left\{\rho_{A_{1} \ldots A_{m} C}^{n}\right\}$ converging to a state $\rho_{A_{1} \ldots A_{m} C}^{0}$ and any sequence $\left\{\Phi_{C}^{n}\right\} \subset \mathfrak{F}\left(C, C^{\prime}\right)$ strongly converging to a channel $\Phi_{C}^{0}$ provided that the Choi rank of all the channels $\Phi_{C}^{n}$ does not exceed $k$ and $I\left(A_{1}: \ldots: A_{m} \mid C\right)_{\rho^{n}}<+\infty$ for all $n \geq 0$.

\section{Applications}

\subsection{Continuity conditions for QCMI}

\subsubsection{Tripartite system}

Theorem 2 implies, by Lemma 2, the following observations that can be interpreted as preserving of local continuity of QCMI under action of strongly converging sequences of local channels.

Proposition 2. If $\left\{\rho_{A B C}^{n}\right\}$ is a sequence of states converging to a state $\rho_{A B C}^{0}$ such that $\lim _{n \rightarrow+\infty} I(A: B \mid C)_{\rho^{n}}=I(A: B \mid C)_{\rho^{0}}<+\infty$ then

$$
\lim _{n \rightarrow+\infty} I\left(A^{\prime}: B^{\prime} \mid C\right)_{\Phi_{A}^{n} \otimes \Phi_{B}^{n} \otimes \operatorname{Id}_{C}\left(\rho^{n}\right)}=I\left(A^{\prime}: B^{\prime} \mid C\right)_{\Phi_{A}^{0} \otimes \Phi_{B}^{0} \otimes \operatorname{Id}_{C}\left(\rho^{0}\right)}
$$

for arbitrary sequences $\left\{\Phi_{A}^{n}\right\} \subset \mathfrak{F}\left(A, A^{\prime}\right)$ and $\left\{\Phi_{B}^{n}\right\} \subset \mathfrak{F}\left(B, B^{\prime}\right)$ strongly converging to channels $\Phi_{A}^{0}$ and $\Phi_{B}^{0}$ correspondingly 8

If, in addition, $\lim _{n \rightarrow+\infty} H\left(\rho_{C}^{n}\right)=H\left(\rho_{C}^{0}\right)<+\infty$ then

$$
\lim _{n \rightarrow+\infty} I\left(A^{\prime}: B^{\prime} \mid C^{\prime}\right)_{\Phi_{A}^{n} \otimes \Phi_{B}^{n} \otimes \Phi_{C}^{n}\left(\rho^{n}\right)}=I\left(A^{\prime}: B^{\prime} \mid C^{\prime}\right)_{\Phi_{A}^{0} \otimes \Phi_{B}^{0} \otimes \Phi_{C}^{0}\left(\rho^{0}\right)}
$$

for arbitrary sequences $\left\{\Phi_{A}^{n}\right\} \subset \mathfrak{F}\left(A, A^{\prime}\right),\left\{\Phi_{B}^{n}\right\} \subset \mathfrak{F}\left(B, B^{\prime}\right)$ and $\left\{\Phi_{C}^{n}\right\} \subset \mathfrak{F}\left(C, C^{\prime}\right)$ strongly converging to channels $\Phi_{A}^{0}, \Phi_{B}^{0}$ and $\Phi_{C}^{0}$. The condition $\lim _{n \rightarrow+\infty} H\left(\rho_{C}^{n}\right)=$ $H\left(\rho_{C}^{0}\right)<+\infty$ can be omitted if all the channels $\Phi_{C}^{n}$ have bounded Choi rank.

To illustrate Proposition 2 consider the following

Example 1. Let $\rho_{A B C}$ be a state such that $I(A: B \mid C)_{\rho}$ and $H\left(\rho_{C}\right)$ are finite. Let $\left\{\Phi_{A}^{t}: A \rightarrow A\right\}_{t \in \mathbb{R}_{+}},\left\{\Phi_{B}^{t}: B \rightarrow B\right\}_{t \in \mathbb{R}_{+}}$and $\left\{\Phi_{C}^{t}: C \rightarrow C\right\}_{t \in \mathbb{R}_{+}}$be arbitrary strongly continuous families of quantum channels (for instance, quantum dynamical semigroups). Proposition 2 and Theorem 2 imply that the function

$$
t \mapsto I(A: B \mid C)_{\Phi_{A}^{t} \otimes \Phi_{B}^{t} \otimes \Phi_{C}^{t}(\rho)}
$$

is continuous on $\mathbb{R}_{+}$and bounded above by $I(A: B \mid C)_{\rho}+2 H\left(\rho_{C}\right)$.

\footnotetext{
${ }^{8} \mathfrak{F}\left(X, X^{\prime}\right)$ is the set of all quantum channels from a system $X$ to a system $X^{\prime}$ equipped with the strong convergence topology (see Section 2).
} 
By using Corollary 1 and taking partial traces in the role of the channels $\Phi_{A}^{n}$ and $\Phi_{B}^{n}$ we obtain from Proposition 2 the following condition for local continuity of QCMI.

Corollary 3. Let $\left\{\rho_{A B C}^{n}\right\}$ be a sequence of states converging to a state $\rho_{A B C}^{0}$. If there exist a system $E$ and a sequence $\left\{\hat{\rho}_{A B C E}^{n}\right\}$ of states converging to a state $\hat{\rho}_{A B C E}^{0}$ such that $\hat{\rho}_{A B C}^{n}=\rho_{A B C}^{n}$ for all $n \geq 0$ and $\lim _{n \rightarrow \infty} H\left(\rho_{X}^{n}\right)=H\left(\rho_{X}^{0}\right)<+\infty$, where $X$ is one of the systems $A E, B E, A C E$ and $B C E$, then

$$
\lim _{n \rightarrow \infty} I(A: B \mid C)_{\rho^{n}}=I(A: B \mid C)_{\rho^{0}}<+\infty .
$$

The conditions for local continuity of QCMI given by Corollary 3 is more powerful than the conditions given by Corollary 1 (which corresponds to Corollary 3 with trivial system $E)$. To show this it suffices to consider the case $I(A: B \mid C)=I(A: B)$. In this case Corollary 3 states that $I(A: B)_{\rho^{n}}$ tends to $I(A: B)_{\rho^{0}}$ for a sequence $\left\{\rho_{A B}^{n}\right\}$ converging to a state $\rho_{A B}^{0}$ provided that

$$
\exists\left\{\hat{\rho}_{A B E}^{n}\right\} \rightarrow \hat{\rho}_{A B E}^{0} \quad \text { such that } \quad \hat{\rho}_{A B}^{n}=\rho_{A B}^{n} \forall n \geq 0 \quad \text { and } \quad H\left(\hat{\rho}_{X}^{n}\right) \rightarrow H\left(\hat{\rho}_{X}^{0}\right),
$$

where $X$ is either $A E$ or $B E$. So, to prove continuity of $I(A: B)$ for a given converging sequence $\left\{\rho_{A B}^{n}\right\}$ it suffices, briefly speaking, to find a converging sequence $\left\{\hat{\rho}_{A B E}^{n}\right\}$ of extensions such that either $H\left(\hat{\rho}_{A E}^{n}\right)$ or $H\left(\hat{\rho}_{B E}^{n}\right)$ is continuous. The following example demonstrates how to use this condition.

Example 2. Let

$$
\rho_{A B}^{n}=\sum_{i=1}^{d} p_{i}^{n} \alpha_{i}^{n} \otimes \beta_{i}^{n}, \quad n=0,1,2, \ldots
$$

where $d \leq+\infty, \alpha_{i}^{n} \in \mathfrak{S}\left(\mathcal{H}_{A}\right)$ and $\beta_{i}^{n} \in \mathfrak{S}\left(\mathcal{H}_{B}\right)$ for all $i$ and $n$ and $\left\{p_{i}^{n}\right\}_{i=1}^{d}$ is a probability distribution for each $n \geq 0$. Assume that

$$
\lim _{n \rightarrow+\infty} \alpha_{i}^{n}=\alpha_{i}^{0}, \quad \lim _{n \rightarrow+\infty} \beta_{i}^{n}=\beta_{i}^{0} \quad \text { and } \quad \lim _{n \rightarrow+\infty} p_{i}^{n}=p_{i}^{0} \quad \text { for each } i .
$$

By using Lemma 1 and its classical counterpart it is easy to show that the sequence $\left\{\rho_{A B}^{n}\right\}$ tends to the state $\rho_{A B}^{0}$.

Let $\mathcal{H}_{E}=\bigoplus_{i=1}^{d} \mathcal{H}_{E_{i}}$, where $\mathcal{H}_{E_{i}}$ is a separable Hilbert space for any $i$. For each $i$ let $\left\{\hat{\alpha}_{i}^{n}\right\}_{n}$ be a sequence of pure states in $\mathcal{H}_{A E_{i}}$ converging to a pure state $\hat{\alpha}_{i}^{0}$ such that $\operatorname{Tr}_{E_{i}} \hat{\alpha}_{i}^{n}=\alpha_{i}^{n}$ for all $n \geq 0$. By using Lemma 1 it is easy to show that

$$
\hat{\rho}_{A B E}^{n}=\sum_{i=1}^{d} p_{i}^{n} \hat{\alpha}_{i}^{n} \otimes \beta_{i}^{n} \rightarrow \hat{\rho}_{A B E}^{0}=\sum_{i=1}^{d} p_{i}^{0} \hat{\alpha}_{i}^{0} \otimes \beta_{i}^{0} \quad \text { as } \quad n \rightarrow+\infty .
$$

It is clear that $\hat{\rho}_{A B}^{n}=\rho_{A B}^{n}$ for all $n \geq 0$. Since for each $n$ all the pure states $\hat{\alpha}_{1}^{n}, \hat{\alpha}_{2}^{n}, \ldots$ are mutually orthogonal, $H\left(\hat{\rho}_{A E}^{n}\right)=S\left(\left\{p_{i}^{n}\right\}\right)$ - the Shannon entropy of the probability distribution $\left\{p_{i}^{n}\right\}$. Thus, condition (30) implies that

$$
\lim _{n \rightarrow+\infty} I(A: B)_{\rho^{n}}=I(A: B)_{\rho^{0}}<+\infty \text { provided that } \lim _{n \rightarrow+\infty} S\left(\left\{p_{i}^{n}\right\}\right)=S\left(\left\{p_{i}^{0}\right\}\right)<+\infty \text {. }
$$


In particular, if $d<+\infty$ then $I(A: B)_{\rho^{n}}$ always tends to $I(A: B)_{\rho^{0}}$.

It looks natural (keeping in mind the physical sense of $I(A: B)$ ) that the above continuity condition for $I(A: B)_{\rho^{n}}$ does not depend on properties of the states $\alpha_{i}^{n}$ and $\beta_{i}^{n}$, in particular, on their entropies. Note that this condition can not be obtained from Corollary 1, i.e. without construction of appropriate extension of the sequence $\left\{\rho_{A B}^{n}\right\}$. Moreover, if $H\left(\alpha_{i_{1}}^{0}\right)=H\left(\beta_{i_{2}}^{0}\right)=+\infty$ for some $i_{1}$ and $i_{2}$ then Corollary 1 can say nothing about the convergence of $I(A: B)_{\rho^{n}}$ to $I(A: B)_{\rho^{0}}$ for the above sequence of states.

Since we have not found a sequence $\left\{\rho_{A B}^{n}\right\}$ converging to a state $\rho_{A B}^{0}$ such that

$$
\lim _{n \rightarrow+\infty} I(A: B)_{\rho^{n}}=I(A: B)_{\rho^{0}}<+\infty
$$

but condition (30) is not valid, it is reasonable to propose the following

Conjecture. Condition (30) is equivalent to (31) 9

To prove this conjecture it is sufficient to show that for any sequence $\left\{\rho_{A B}^{n}\right\}$ converging to a state $\rho_{A B}^{0}$ such that (31) holds there is a sequence $\left\{\hat{\rho}_{A A^{\prime} B B^{\prime}}^{n}\right\}$ of pure states converging to a pure state $\hat{\rho}_{A A^{\prime} B B^{\prime}}^{0}$ such that

$$
\lim _{n \rightarrow+\infty} I\left(A A^{\prime}: B B^{\prime}\right)_{\hat{\rho}^{n}}=I\left(A A^{\prime}: B B^{\prime}\right)_{\hat{\rho}^{0}}<+\infty
$$

\subsubsection{Multipartite system}

The following multipartite version of Proposition 2 is obtained from Theorem 3 by means of Lemma 2.

Proposition 3. If $\left\{\rho_{A_{1} \ldots A_{m} C}^{n}\right\}$ is a sequence of states converging to a state $\rho_{A_{1} \ldots A_{m} C}^{0}$ such that

$$
\lim _{n \rightarrow+\infty} I\left(A_{1}: \ldots: A_{m} \mid C\right)_{\rho^{n}}=I\left(A_{1}: \ldots: A_{m} \mid C\right)_{\rho^{0}}<+\infty
$$

then

$$
\lim _{n \rightarrow+\infty} I\left(A_{1}^{\prime}: \ldots: A_{m}^{\prime} \mid C\right)_{\Phi_{A_{1}}^{n} \otimes \ldots \otimes \Phi_{A_{m}}^{n} \otimes \operatorname{Id}_{C}\left(\rho^{n}\right)}=I\left(A_{1}^{\prime}: \ldots: A_{m}^{\prime} \mid C\right)_{\Phi_{A_{1}}^{0} \otimes \ldots \otimes \Phi_{A_{m}}^{0} \otimes \operatorname{Id}_{C}\left(\rho^{0}\right)}
$$

for arbitrary sequences $\left\{\Phi_{A_{1}}^{n}\right\} \subset \mathfrak{F}\left(A_{1}, A_{1}^{\prime}\right), \ldots,\left\{\Phi_{A_{m}}^{n}\right\} \subset \mathfrak{F}\left(A_{m}, A_{m}^{\prime}\right)$ strongly converging to channels $\Phi_{A_{1}}^{0}, \ldots, \Phi_{A_{m}}^{0}$ correspondingly.

If, in addition, $\lim _{n \rightarrow+\infty} H\left(\rho_{C}^{n}\right)=H\left(\rho_{C}^{0}\right)<+\infty$ then

$$
\lim _{n \rightarrow+\infty} I\left(A_{1}^{\prime}: \ldots: A_{m}^{\prime} \mid C^{\prime}\right)_{\Phi_{A_{1}}^{n} \otimes \ldots \otimes \Phi_{A_{m}}^{n} \otimes \Phi_{C}^{n}\left(\rho^{n}\right)}=I\left(A_{1}^{\prime}: \ldots: A_{m}^{\prime} \mid C^{\prime}\right)_{\Phi_{A_{1}}^{0} \otimes \ldots \otimes \Phi_{A_{m}}^{0} \otimes \Phi_{C}^{0}\left(\rho^{0}\right)}
$$

for arbitrary sequences $\left\{\Phi_{A_{1}}^{n}\right\} \subset \mathfrak{F}\left(A_{1}, A_{1}^{\prime}\right), \ldots,\left\{\Phi_{A_{m}}^{n}\right\} \subset \mathfrak{F}\left(A_{m}, A_{m}^{\prime}\right)$ and $\left\{\Phi_{C}^{n}\right\} \subset \mathfrak{F}\left(C, C^{\prime}\right)$ strongly converging to channels $\Phi_{A_{1}}^{0}, \ldots, \Phi_{A_{m}}^{0}$ and $\Phi_{C}^{0}$ correspondingly. The condition $\lim _{n \rightarrow+\infty} H\left(\rho_{C}^{n}\right)=H\left(\rho_{C}^{0}\right)<+\infty$ can be omitted if all the channels $\Phi_{C}^{n}$ have bounded Choi rank.

\footnotetext{
${ }^{9}$ I would be grateful for any comments concerning this conjecture.
} 
By Proposition 5D in [29] relation (32) holds if there is a set of indexes $i_{1}, \ldots, i_{m-1}$ s.t. $\lim _{n \rightarrow \infty} H\left(\rho_{X_{i_{k}}}^{n}\right)=H\left(\rho_{X_{i_{k}}}^{0}\right)<+\infty, k=\overline{1, m-1}$, where $X_{i_{k}}$ is either $A_{i_{k}}$ or $A_{i_{k}} C$.

By taking partial traces in the role of the channels $\Phi_{A_{i}}^{n}$ in Proposition 3 one can strengthen the above condition as follows.

Corollary 4. Let $\left\{\rho_{A_{1} \ldots A_{m} C}^{n}\right\}$ be a sequence of states converging to a state $\rho_{A_{1} \ldots A_{m} C}^{0}$. If there exist systems $E_{1}, \ldots E_{m-1}$ and a sequence $\left\{\hat{\rho}_{A_{1} \ldots A_{m} C E_{1} \ldots E_{m-1}}^{n}\right\}$ of states converging to a state $\hat{\rho}_{A_{1} \ldots A_{m} C E_{1} \ldots E_{m-1}}^{0}$ such that $\hat{\rho}_{A_{1} \ldots A_{m} C}^{n}=\rho_{A_{1} \ldots A_{m} C}^{n}$ for all $n \geq 0$ and $\lim _{n \rightarrow+\infty} H\left(\rho_{X_{i_{k}}}^{n}\right)=H\left(\rho_{X_{i_{k}}}^{0}\right)<+\infty$ for some set of indexes $i_{1}, \ldots, i_{m-1}$, where $X_{i_{k}}$ is either $A_{i_{k}} E_{k}$ or $A_{i_{k}} C E_{k}$, for each $k=\overline{1, m-1}$, then relation (32) holds.

Corollary 4 is a $m$-partite version of Corollary 3. It allows to prove relation (32) for a converging sequence of states by constructing appropriate extension of this sequence.

Let $\left\{\rho_{A_{1} \ldots A_{m}}^{n}\right\}$ be a natural $m$-partite generalization of the converging sequence of separable states from the above Example 1. By the similar way one can construct a sequence $\left\{\hat{\rho}_{A_{1} \ldots A_{m} E_{1} \ldots E_{m-1}}^{n}\right\}$ of extensions converging to an extension $\left\{\hat{\rho}_{A_{1} \ldots A_{m} E_{1} \ldots E_{m-1}}^{0}\right\}$ of the state $\rho_{A_{1} \ldots A_{m}}^{0}$ such that for $k=\overline{1, m-1}$ and all $n \geq 0$ the von Neumann entropy $H\left(\rho_{A_{k} E_{k}}^{n}\right)$ coincides with the Shannon entropy $S\left(\left\{p_{i}^{n}\right\}\right)$ of the probability distribution from the decomposition of $\rho_{A_{1} \ldots A_{m}}^{n}$ into product states. Thus, Corollary 4 allows to show that

$$
\lim _{n \rightarrow+\infty} S\left(\left\{p_{i}^{n}\right\}\right)=S\left(\left\{p_{i}^{0}\right\}\right)<+\infty \Rightarrow \lim _{n \rightarrow+\infty} I\left(A_{1}: \ldots: A_{m}\right)_{\rho^{n}}=I\left(A_{1}: \ldots: A_{m}\right)_{\rho^{0}}
$$

regardless of characteristics of the product states involved in the decompositions of the states $\rho_{A_{1} \ldots A_{m}}^{n}$. Note that this assertion can not be proved by using the conditions for local continuity of $I\left(A_{1}: \ldots: A_{m}\right)$ existing in the literature (in particular, by using Proposition 5D in [29] mentioned before).

\subsection{Continuity conditions for the squashed entanglement}

\subsubsection{Bipartite system}

The squashed entanglement is one of the basic entanglement measures defined for a state $\rho_{A B}$ of a finite-dimensional bipartite system $A B$ as

$$
E_{s q}\left(\rho_{A B}\right)=\frac{1}{2} \inf _{\rho_{A B E}} I(A: B \mid E)
$$

where the infimum is over all extensions $\rho_{A B E}$ of the state $\rho_{A B}$ [6, 35]. By using the extended QCMI defined by the equivalent formulae (17) and (18) this definition can be generalized for any state $\rho_{A B}$ of an infinite-dimensional bipartite system $A B$. It is shown in [30] that in this case the function $E_{s q}$ defined in (33) possesses all basic properties of an entanglement measure (cf.[18, 27]) excepting the global continuity and the vanishing on the set of all separable states (the latter property is conjectured but not proved because of the existence of separable states that are not countablydecomposable, see Remark 10 in [30]). It is also shown that the function $E_{s q}$ is lower 
semicontinuous on the set

$$
\mathfrak{S}_{*}\left(\mathcal{H}_{A B}\right) \doteq\left\{\rho_{A B} \mid \min \left\{H\left(\rho_{A}\right), H\left(\rho_{B}\right), H\left(\rho_{A B}\right)\right\}<+\infty\right\}
$$

and coincides on this set with the convex closure of $E_{s q}$ - the maximal convex lower semicontinuous function on $\mathfrak{S}\left(\mathcal{H}_{A B}\right)$ not exceeding $E_{s q} 10$ This implies, in particular, that $E_{s q}\left(\rho_{A B}\right)=0$ for any separable state $\rho_{A B}$ belonging to the set $\mathfrak{S}_{*}\left(\mathcal{H}_{A B}\right)$ [30].

A special attention is paid in [30] to analysis of local continuity of $E_{s q}$. It is proved that $\lim _{n \rightarrow+\infty} E_{s q}\left(\rho_{A B}^{n}\right)=E_{s q}\left(\rho_{A B}^{0}\right)$ for a sequence $\left\{\rho_{A B}^{n}\right\}$ converging to a state $\rho_{A B}^{0}$ provided that

$$
\lim _{n \rightarrow+\infty} I(A: B)_{\rho^{n}}=I(A: B)_{\rho^{0}}<+\infty \quad \text { and } \min \left\{H\left(\rho_{A}^{0}\right), H\left(\rho_{B}^{0}\right)\right\}<+\infty .
$$

By Theorem 1 in [29] this condition holds if either

$$
\lim _{n \rightarrow+\infty} H\left(\rho_{A}^{n}\right)=H\left(\rho_{A}^{0}\right)<+\infty \quad \text { or } \quad \lim _{n \rightarrow+\infty} H\left(\rho_{B}^{n}\right)=H\left(\rho_{B}^{0}\right)<+\infty .
$$

By combining Proposition 2 in Section 5.1.1 with Proposition 13 in [30] we obtain the following

Proposition 4. Let $\left\{\rho_{A B}^{n}\right\}$ be a sequence of states converging to a state $\rho_{A B}^{0}$. Then

$$
\lim _{n \rightarrow+\infty} I(A: B)_{\rho^{n}}=I(A: B)_{\rho^{0}}<+\infty \Rightarrow \lim _{n \rightarrow+\infty} E_{s q}\left(\Phi_{A}^{n} \otimes \Phi_{B}^{n}\left(\rho_{A B}^{n}\right)\right)=E_{s q}\left(\Phi_{A}^{0} \otimes \Phi_{B}^{0}\left(\rho_{A B}^{0}\right)\right)
$$

for arbitrary sequences $\left\{\Phi_{A}^{n}\right\} \subset \mathfrak{F}\left(A, A^{\prime}\right)$ and $\left\{\Phi_{B}^{n}\right\} \subset \mathfrak{F}\left(B, B^{\prime}\right)$ strongly converging to channels $\Phi_{A}^{0}$ and $\Phi_{B}^{0}$ provided that either $H\left(\Phi_{A}^{0}\left(\rho_{A}^{0}\right)\right)$ or $H\left(\Phi_{B}^{0}\left(\rho_{B}^{0}\right)\right)$ is finite 11

By using Corollary 1 and taking partial traces in the role of the channels $\Phi_{A}^{n}$ and $\Phi_{B}^{n}$ we obtain from Proposition 4 the following condition for local continuity of $E_{s q}$.

Corollary 5. Let $\left\{\rho_{A B}^{n}\right\}$ be a sequence converging to a state $\rho_{A B}^{0}$ such that either $H\left(\rho_{A}^{0}\right)$ or $H\left(\rho_{B}^{0}\right)$ is finite. If there exist a system $E$ and a sequence $\left\{\hat{\rho}_{A B E}^{n}\right\}$ converging to a state $\hat{\rho}_{A B E}^{0}$ such that $\hat{\rho}_{A B}^{n}=\rho_{A B}^{n}$ for all $n \geq 0$ and $\lim _{n \rightarrow+\infty} H\left(\rho_{X}^{n}\right)=H\left(\rho_{X}^{0}\right)<+\infty$, where $X$ is either $A E$ or $B E$, then

$$
\lim _{n \rightarrow+\infty} E_{s q}\left(\rho_{A B}^{n}\right)=E_{s q}\left(\rho_{A B}^{0}\right)<+\infty .
$$

The assertion of Corollary 5 can be also obtained by combining condition (34) with the continuity condition (30) for the quantum mutual information.

The continuity condition given by Corollary 5 can be treated as a strengthened form of condition (35). To show superiority (and applicability) of the former condition consider the following

\footnotetext{
${ }^{10}$ It is shown in $\left[30\right.$ that the convex closure of $E_{s q}$ can be obtained from the finite-dimensional squashed entanglement by the construction called universal extension. These is a conjecture that $E_{s q}$ coincides with its convex closure on the whole space $\mathfrak{S}\left(\mathcal{H}_{A B}\right)$.

${ }^{11} \mathfrak{F}\left(X, X^{\prime}\right)$ is the set of all quantum channels from a system $X$ to a system $X^{\prime}$ equipped with the strong convergence topology (see Section 2).
} 
Example 3. Let $\left\{\rho_{A}^{n}\right\},\left\{\rho_{B}^{n}\right\}$ and $\left\{\sigma_{A B}^{n}\right\}$ be sequences of states converging, respectively, to states $\rho_{A}^{0}, \rho_{B}^{0}$ and $\sigma_{A B}^{0}$. Corollary 5 allows to show that

$$
\lim _{n \rightarrow+\infty} E_{s q}\left((1-p) \rho_{A}^{n} \otimes \rho_{B}^{n}+p \sigma_{A B}^{n}\right)=E_{s q}\left((1-p) \rho_{A}^{0} \otimes \rho_{B}^{0}+p \sigma_{A B}^{0}\right), \quad p \in[0,1],
$$

provided that

$$
\lim _{n \rightarrow+\infty} H\left(\sigma_{X}^{n}\right)=H\left(\sigma_{X}^{0}\right)<+\infty
$$

and $H\left(\rho_{X}^{0}\right)<+\infty$, where $X$ is either $A$ or $B$, regardless of the entropies of the states $\rho_{A}^{n}$ and $\rho_{B}^{n}$. Indeed, assume that $X=A$ and $\left\{\hat{\rho}_{A E}^{n}\right\}$ is a sequence of pure states converging to a pure state $\hat{\rho}_{A E}^{0}$ such that $\hat{\rho}_{A}^{n}=\rho_{A}^{n}$ for all $n \geq 0$. Let $\tau_{E}$ be a pure state orthogonal to all the states $\hat{\rho}_{E}^{n}$ (we may always extend the system $E$ if necessary). Then the sequence of states

$$
\varrho_{A B E}^{n}=(1-p) \hat{\rho}_{A E}^{n} \otimes \rho_{B}^{n}+p \sigma_{A B}^{n} \otimes \tau_{E}
$$

tends to the state $\varrho_{A B E}^{0}=(1-p) \hat{\rho}_{A E}^{0} \otimes \rho_{B}^{0}+p \sigma_{A B}^{0} \otimes \tau_{E}, \varrho_{A B}^{n}=(1-p) \rho_{A}^{n} \otimes \rho_{B}^{n}+p \sigma_{A B}^{n}$ and $H\left(\varrho_{A E}^{n}\right)=p H\left(\sigma_{A}^{n}\right)+h_{2}(p)$ for all $n \geq 0$. Thus, the assumption (36) with $X=A$ implies that

$$
\lim _{n \rightarrow+\infty} H\left(\varrho_{A E}^{n}\right)=H\left(\varrho_{A E}^{0}\right)<+\infty .
$$

By considering the case when $p=1$ and all the states $\sigma_{A B}^{n}$ are pure we see that condition (36) is essential. Note that the use of condition (35) for the above sequence of state leads to the additional "nonphysical" requirement

$$
\lim _{n \rightarrow+\infty} H\left(\rho_{X}^{n}\right)=H\left(\rho_{X}^{0}\right)<+\infty .
$$

The above example can be generalized by considering the sequences of the form

$$
(1-p) \rho_{A B}^{n}+p \sigma_{A B}^{n},
$$

where $\left\{\rho_{A B}^{n}\right\}$ is the converging sequence of separable states from Example 2 .

\subsubsection{Multipartite system}

The squashed entanglement of a state $\rho_{A_{1} \ldots A_{m}}$ of a finite-dimensional composite system $A_{1} \ldots A_{m}$ is defined as

$$
E_{s q}\left(\rho_{A_{1} \ldots A_{m}}\right)=\frac{1}{2} \inf _{\rho_{A_{1} \ldots A_{m} E}} I\left(A_{1}: \ldots: A_{m} \mid E\right)_{\rho}
$$

where the infimum is over all extensions $\rho_{A_{1} \ldots A_{m} E}$ of the state $\rho_{A_{1} \ldots A_{m}}$ [1, 41, 12 By using the extended multipartite QCMI described in Section 3.2 this definition can be

\footnotetext{
${ }^{12}$ In [1, 41] two $m$-partite generalizations of the bipartite squashed entanglement are proposed: the first one is defined in (37), the second one is defined by the expression similar to (37) with the different $m$-partite version of QCMI (called dual conditional total correlation). In [9] it is proved that these $m$-partite generalizations of the bipartite squashed entanglement coincide.
} 
generalized for any state $\rho_{A_{1} \ldots A_{m}}$ of an infinite-dimensional composite system $A_{1} \ldots A_{m}$. By using the arguments from [1, 41] one can show that in this case the function $E_{s q}$ defined by formula (37) possesses almost all properties of an entanglement measure, in particular, it is convex on the whole set of states of an infinite-dimensional composite system $A_{1} \ldots A_{m}$ and nonincreasing under LOCC. Similar to the bipartite case, it is not clear how to show that $E_{s q}$ is equal to zero on the set of all separable states because of the existence of countably nondecomposable separable states in infinite-dimensional composite systems (see Remark 10 in [30]). Below we show that $E_{s q}$ is equal to zero on the set of all separable states in $\mathfrak{S}\left(\mathcal{H}_{A_{1} \ldots A_{m}}\right)$ with finite marginal entropies (Corollary 6) by using the following proposition describing continuity properties of $E_{s q}$ in infinite dimensions.

Proposition 5. A) The function $E_{s q}\left(\rho_{A_{1} \ldots A_{m}}\right)$ is lower semicontinuous on the set

$$
\mathfrak{S}_{*}\left(\mathcal{H}_{A_{1} \ldots A_{m}}\right) \doteq\left\{\rho_{A_{1} \ldots A_{m}} \mid H\left(\rho_{A_{1}}\right), \ldots, H\left(\rho_{A_{m}}\right)<+\infty\right\} .
$$

B) If $\left\{\rho_{A_{1} \ldots A_{m}}^{n}\right\}$ is a sequence of states converging to a state $\rho_{A_{1} \ldots A_{m}}^{0}$ such that

$$
\lim _{n \rightarrow+\infty} I\left(A_{1}: \ldots: A_{m}\right)_{\rho^{n}}=I\left(A_{1}: \ldots: A_{m}\right)_{\rho^{0}}<+\infty
$$

and $\rho_{A_{1} \ldots A_{m}}^{0} \in \mathfrak{S}_{*}\left(\mathcal{H}_{A_{1} \ldots A_{m}}\right)$ then

$$
\lim _{n \rightarrow+\infty} E_{s q}\left(\rho_{A_{1} \ldots A_{m}}^{n}\right)=E_{s q}\left(\rho_{A_{1} \ldots A_{m}}^{0}\right) .
$$

Moreover, for arbitrary sequences $\left\{\Phi_{A_{1}}^{n}\right\} \subset \mathfrak{F}\left(A_{1}, A_{1}^{\prime}\right), \ldots,\left\{\Phi_{A_{m}}^{n}\right\} \subset \mathfrak{F}\left(A_{m}, A_{m}^{\prime}\right)$ strongly converging to channels $\Phi_{A_{1}}^{0}, \ldots, \Phi_{A_{m}}^{0}$ such that all the states $\Phi_{A_{1}}^{0}\left(\rho_{A_{1}}^{0}\right), \ldots, \Phi_{A_{m}}^{0}\left(\rho_{A_{m}}^{0}\right)$ have finite entropy condition (39) implies that

$$
\lim _{n \rightarrow+\infty} E_{s q}\left(\Phi_{A_{1}}^{n} \otimes \ldots \otimes \Phi_{A_{m}}^{n}\left(\rho_{A_{1} \ldots A_{m}}^{n}\right)\right)=E_{s q}\left(\Phi_{A_{1}}^{0} \otimes \ldots \otimes \Phi_{A_{m}}^{0}\left(\rho_{A_{1} \ldots A_{m}}^{0}\right)\right) .
$$

Proof. A) Let $\left\{P_{1}^{n}\right\} \subset \mathfrak{B}\left(\mathcal{H}_{A_{1}}\right), \ldots,\left\{P_{m}^{n}\right\} \subset \mathfrak{B}\left(\mathcal{H}_{A_{m}}\right)$ be sequences of finite rank projectors strongly converging to the unit operators $I_{A_{1}}, \ldots, I_{A_{m}}$ such that $\operatorname{rank} P_{k}^{n} \leq n$, $k=\overline{1, m}$. For each $k$ and $n$ consider the channel

$$
\Phi_{k}^{n}(\varrho)=P_{k}^{n} \varrho P_{k}^{n}+\tau_{k}^{n}\left[\operatorname{Tr}\left(I_{A_{k}}-P_{k}^{n}\right) \varrho\right],
$$

from $\mathfrak{S}\left(\mathcal{H}_{A_{k}}\right)$ to itself, where $\tau_{k}^{n}$ is a pure state supported by the range of $P_{k}^{n}$. It is easy to see that the sequence $\left\{\Phi_{k}^{n}\right\}_{n}$ strongly converges to the identity channel $\operatorname{Id}_{A_{k}}$.

For given $n$ let

$$
f_{n}\left(\rho_{A_{1} \ldots A_{m}}\right)=\frac{1}{2} \inf _{\rho_{A_{1} \ldots A_{m} E}} I\left(A_{1}: \ldots: A_{m} \mid E\right)_{\Phi_{1}^{n} \otimes \ldots \otimes \Phi_{m}^{n} \otimes \operatorname{Id}_{E}(\rho)}
$$

where the infimum is over all extensions $\rho_{A_{1} \ldots A_{m} E}$ of the state $\rho_{A_{1} \ldots A_{m}}$. We will show that the function $f_{n}$ is continuous on the space $\mathfrak{S}\left(\mathcal{H}_{A_{1}, ., A_{m}}\right)$ for each $n$. 
Let $\rho_{A_{1} \ldots A_{m}}^{1}$ and $\rho_{A_{1} \ldots A_{m}}^{2}$ be states such that $\left\|\rho_{A_{1} \ldots A_{m}}^{1}-\rho_{A_{1} \ldots A_{m}}^{2}\right\|_{1} \leq \varepsilon$. By the arguments from the proof of continuity of $E_{s q}$ in [6] we have

$$
f_{n}\left(\rho_{A_{1} \ldots A_{m}}^{t}\right)=\frac{1}{2} \inf _{\Lambda} I\left(A_{1}: \ldots: A_{m} \mid E\right)_{\Phi_{1}^{n} \otimes \ldots \otimes \Phi_{m}^{n} \otimes \mathrm{I}_{E}\left(\Lambda\left(\hat{\rho}^{t}\right)\right)}, \quad t=1,2,
$$

where $\hat{\rho}_{A_{1} \ldots A_{m} R}^{1}$ and $\hat{\rho}_{A_{1} \ldots A_{m} R}^{2}$ are purifications of the states $\rho_{A_{1} \ldots A_{m}}^{1}$ and $\rho_{A_{1} \ldots A_{m}}^{2}$ such that

$$
\left\|\hat{\rho}_{A_{1} \ldots A_{m} R}^{1}-\hat{\rho}_{A_{1} \ldots A_{m} R}^{2}\right\|_{1} \leq 2 \sqrt{\varepsilon}
$$

and the infimum is over all "squashing" channels $\Lambda: R \rightarrow E$.

For a given channel $\Lambda$ let $\sigma_{A_{1} \ldots A_{m} E}^{t}=\Phi_{1}^{n} \otimes \ldots \otimes \Phi_{m}^{n} \otimes \operatorname{Id}_{E}\left(\Lambda\left(\hat{\rho}_{A_{1} \ldots A_{m} R}\right)\right), t=1,2$. By monotonicity of the trace norm under action of a channel we have

$$
\left\|\sigma_{A_{1} \ldots A_{m} E}^{1}-\sigma_{A_{1} \ldots A_{m} E}^{2}\right\|_{1} \leq 2 \sqrt{\varepsilon}
$$

Since $\operatorname{rank} \sigma_{A_{k}}^{1}, \operatorname{rank} \sigma_{A_{k}}^{2} \leq n$ for all $k$, Proposition $5 \mathrm{E}$ in [29] implies that

$$
\left|I\left(A_{1}: \ldots: A_{m} \mid E\right)_{\sigma^{1}}-I\left(A_{1}: \ldots: A_{m} \mid E\right)_{\sigma^{2}}\right| \leq 2(m-1) \sqrt{\varepsilon} \log n+2 m g(\sqrt{\varepsilon}),
$$

where $g(x)=(x+1) \log (x+1)-x \log x$. It follows that $\left|f_{n}\left(\rho_{A_{1} \ldots A_{m}}^{1}\right)-f_{n}\left(\rho_{A_{1} \ldots A_{m}}^{2}\right)\right| \leq$ $2(m-1) \sqrt{\varepsilon} \log n+2 m g(\sqrt{\varepsilon})$, and hence the function $f_{n}$ is uniformly continuous on $\mathfrak{S}\left(\mathcal{H}_{A_{1}, ., A_{m}}\right)$.

Thus, since $f_{n}\left(\rho_{A_{1} \ldots A_{m}}\right) \leq E_{s q}\left(\rho_{A_{1} \ldots A_{m}}\right)$ for any $\rho_{A_{1} \ldots A_{m}}$ and all $n$ by the monotonicity of QCMI, to prove the lower semicontinuity of $E_{s q}$ on the set $\mathfrak{S}_{*}\left(\mathcal{H}_{A_{1} \ldots A_{m}}\right)$ it suffices to show that $f_{n}\left(\rho_{A_{1} \ldots A_{m}}\right)$ tends to $E_{s q}\left(\rho_{A_{1} \ldots A_{m}}\right)$ as $n \rightarrow+\infty$ for any state $\rho_{A_{1} \ldots A_{m}}$ in $\mathfrak{S}_{*}\left(\mathcal{H}_{A_{1} \ldots A_{m}}\right)$. For a given extension $\rho_{A_{1} \ldots A_{m} E}$ of this state we have

$$
\begin{gathered}
\left|I\left(A_{1}: \ldots: A_{m} \mid E\right)_{\rho}-I\left(A_{1}: \ldots: A_{m} \mid E\right)_{\Phi_{1}^{n} \otimes \ldots \otimes \Phi_{m}^{n} \otimes \operatorname{Id}_{E}(\rho)}\right| \\
\leq \sum_{k=1}^{m}\left|I\left(A_{1}: \ldots: A_{m} \mid E\right)_{\Psi_{k-1}^{n} \otimes \operatorname{Id}_{E}(\rho)}-I\left(A_{1}: \ldots: A_{m} \mid E\right)_{\Psi_{k}^{n} \otimes \operatorname{Id}_{E}(\rho)}\right|,
\end{gathered}
$$

where $\Psi_{k}^{n}=\Phi_{1}^{n} \otimes \ldots \otimes \Phi_{k}^{n} \otimes \operatorname{Id}_{A_{k+1} . . A_{m}}, k=\overline{1, m}$, and $\Psi_{0}^{n}=\operatorname{Id}_{A_{1} . . A_{m}}$. Lemma 4 below implies that

$$
\begin{aligned}
& \left|I\left(A_{1}: \ldots: A_{m} \mid E\right)_{\Psi_{k-1}^{n} \otimes \operatorname{Id}_{E}(\rho)}-I\left(A_{1}: \ldots: A_{m} \mid E\right)_{\Psi_{k}^{n} \otimes \operatorname{Id}_{E}(\rho)}\right| \\
& \quad \leq 2\left[H\left(\rho_{A_{k}}\right)-H\left(P_{k}^{n} \rho_{A_{k}} P_{k}^{n}\right)\right]+h_{2}\left(\operatorname{Tr} P_{k}^{n} \rho_{A_{k}}\right),
\end{aligned}
$$

since $\left[\Psi_{k-1}^{n} \otimes \operatorname{Id}_{E}\left(\rho_{A_{1} \ldots A_{m}}\right)\right]_{A_{k}}=\rho_{A_{k}}$ for $k=\overline{1, m}$ and any $n$. Thus, it follows from (42) that

$$
E_{s q}\left(\rho_{A_{1} \ldots A_{m}}\right)-f_{n}\left(\rho_{A_{1} \ldots A_{m}}\right) \leq \sum_{k=1}^{m}\left(2\left[H\left(\rho_{A_{k}}\right)-H\left(P_{k}^{n} \rho_{A_{k}} P_{k}^{n}\right)\right]+h_{2}\left(\operatorname{Tr} P_{k}^{n} \rho_{A_{k}}\right)\right) .
$$

Since $H\left(\rho_{A_{k}}\right)<+\infty$ for all $k$, the r.h.s. of the last inequality tends to zero as $n \rightarrow+\infty$. 
B) Introduce the monotone sequence of functions

$$
E_{s q}^{d}\left(\varrho_{A_{1} \ldots A_{m}}\right)=\frac{1}{2} \inf _{\varrho_{A_{1}} \ldots A_{m} E} I\left(A_{1}: \ldots: A_{n} \mid E\right)_{\varrho}, \quad \operatorname{dim} \mathcal{H}_{E} \leq d,
$$

where the infimum is over all extensions $\varrho_{A_{1} \ldots A_{m} E}$ of the state $\varrho_{A_{1} \ldots A_{m}}$ such that $E$ is a $d$-dimensional system, pointwise converging as $d \rightarrow+\infty$ to the function

$$
E_{s q}^{*}\left(\varrho_{A_{1} \ldots A_{m}}\right)=\frac{1}{2} \inf _{\varrho_{A_{1} \ldots A_{m} E}} I\left(A_{1}: \ldots: A_{n} \mid E\right)_{\varrho}, \quad \operatorname{dim} \mathcal{H}_{E}<+\infty,
$$

where the infimum is over all extensions $\varrho_{A_{1} \ldots A_{m} E}$ of the state $\varrho_{A_{1} \ldots A_{m}}$ such that $E$ is a finite-dimensional system. By direct generalization of the proof of Lemma 16B in [30] based on the relation

$$
I\left(A_{1}: \ldots: A_{m} \mid E\right)-I\left(A_{1}: \ldots: A_{m}\right)=I\left(A_{1} . . A_{m}: E\right)-\sum_{k=1}^{m} I\left(A_{k}: E\right)
$$

it is easy to show that condition (39) implies that

$$
\lim _{n \rightarrow+\infty} E_{s q}^{d}\left(\rho_{A_{1} \ldots A_{m}}^{n}\right)=E_{s q}^{d}\left(\rho_{A_{1} \ldots A_{m}}^{0}\right)
$$

for any $d$, and hence we have

$$
\limsup _{n \rightarrow+\infty} E_{s q}^{*}\left(\rho_{A_{1} \ldots A_{m}}^{n}\right) \leq E_{s q}^{*}\left(\rho_{A_{1} \ldots A_{m}}^{0}\right) .
$$

Introduce also the function

$$
\widehat{E}_{s q}\left(\varrho_{A_{1} \ldots A_{m}}\right)=\sup _{P_{A_{1}}, \ldots, P_{A_{m}}} E_{s q}\left(P_{A_{1}} \otimes \ldots \otimes P_{A_{m}} \cdot \varrho_{A_{1} \ldots A_{m}} \cdot P_{A_{1}} \otimes \ldots \otimes P_{A_{m}}\right)
$$

where the supremum is over all finite-rank projectors $P_{A_{1}} \in \mathfrak{B}\left(\mathcal{H}_{A_{1}}\right), \ldots, P_{A_{m}} \in \mathfrak{B}\left(\mathcal{H}_{A_{m}}\right)$ and it is assumed that $E_{s q}(\sigma)=c E_{s q}(\sigma / c)$ for any positive operator $\sigma$ in $\mathfrak{T}\left(\mathcal{H}_{A_{1} . . A_{m}}\right)$ such that $\operatorname{Tr} \sigma=c>0$. Note that the function $E_{s q}$ in the r.h.s. of (46) is a finitedimensional squashed entanglement for any set of projectors $P_{A_{1}}, \ldots, P_{A_{m}}$.

According to the notations in [30] the function $\widehat{E}_{s q}$ can be called the universal extension of the finite-dimensional multipartite squashed entanglement. By using the arguments from the proof of Proposition 5 in 30 it is easy to show lower semicontinuity and convexity of the function $\widehat{E}_{s q}$ on $\mathfrak{S}\left(\mathcal{H}_{A_{1} . . A_{m}}\right)$ and its monotonicity under selective unilocal operations.

It follows from the definitions that

$$
E_{s q}^{*}\left(\varrho_{A_{1} . . A_{m}}\right) \geq E_{s q}\left(\varrho_{A_{1} . . A_{m}}\right) \geq \widehat{E}_{s q}\left(\varrho_{A_{1} . . A_{m}}\right) \quad \forall \varrho_{A_{1} . . A_{m}} \in \mathfrak{S}\left(\mathcal{H}_{A_{1} . . A_{m}}\right) .
$$

We will show that

$$
E_{s q}^{*}\left(\varrho_{A_{1} . . A_{m}}\right)=E_{s q}\left(\varrho_{A_{1} . . A_{m}}\right)=\widehat{E}_{s q}\left(\varrho_{A_{1} . . A_{m}}\right) \quad \forall \varrho_{A_{1} . . A_{m}} \in \mathfrak{S}_{*}\left(\mathcal{H}_{A_{1} . . A_{m}}\right) .
$$


Take arbitrary $\varepsilon>0$ and an extension $\varrho_{A_{1} . . A_{m} E}^{\varepsilon}$ such that

$$
E_{s q}\left(\varrho_{A_{1} \ldots A_{m}}\right) \geq \frac{1}{2} I\left(A_{1}: \ldots: A_{m} \mid E\right)_{\varrho^{\varepsilon}}-\varepsilon .
$$

Let $\left\{\Phi_{E}^{n}\right\}$ be a sequence of channels from the system $E$ to itself strongly converging to the identity channel $\operatorname{Id}_{E}$ such that the range of $\Phi_{E}^{n}$ is finite-dimensional for each $n$. Since $H\left(\varrho_{A_{k}}^{\varepsilon}\right)=H\left(\varrho_{A_{k}}\right)<+\infty$ for all $k$, Proposition 5D in [29] shows that

$$
\lim _{n \rightarrow+\infty} I\left(A_{1}: \ldots: A_{m} \mid E\right)_{\operatorname{Id}_{A_{1} . . A m} \otimes \Phi_{E}^{n}\left(\varrho^{\varepsilon}\right)}=I\left(A_{1}: \ldots: A_{m} \mid E\right)_{\varrho^{\varepsilon}} .
$$

Since $\frac{1}{2} I\left(A_{1}: \ldots: A_{m} \mid E\right)_{\operatorname{Id}_{A_{1} . . A_{m}} \otimes \Phi_{E}^{n}\left(\varrho^{\varepsilon}\right)} \geq E_{s q}^{*}\left(\varrho_{A_{1} . . A_{m}}\right)$ for all $n$ by definition of $E_{s q}^{*}$, this limit relation and (49) imply the first equality in (48).

Let $\left\{P_{1}^{n}\right\} \subset \mathfrak{B}\left(\mathcal{H}_{A_{1}}\right), \ldots,\left\{P_{m}^{n}\right\} \subset \mathfrak{B}\left(\mathcal{H}_{A_{m}}\right)$ be sequences of finite rank projectors strongly converging to the unit operators $I_{A_{1}}, \ldots, I_{A_{m}}$. For each sufficiently large $n$ let $\varrho_{A_{1} . . A_{m}}^{n}$ be the state proportional to the operator $P_{1}^{n} \otimes \ldots \otimes P_{m}^{n} \cdot \varrho_{A_{1} . . A_{m}} \cdot P_{1}^{n} \otimes \ldots \otimes P_{m}^{n}$. Then the sequence $\left\{\varrho_{A_{1} . . A_{m}}^{n}\right\}$ belongs to the set $\mathfrak{S}_{*}\left(\mathcal{H}_{A_{1} . . A_{m}}\right)$ and converges to the state $\varrho_{A_{1} . . A_{m}} \in \mathfrak{S}_{*}\left(\mathcal{H}_{A_{1} . . A_{m}}\right)$. By part A of this proposition the function $E_{s q}$ is lower semicontinuous on $\mathfrak{S}_{*}\left(\mathcal{H}_{A_{1} . . A_{m}}\right)$. This property and the nonincreasing of $E_{s q}$ under unilocal operations imply that

$$
\lim _{n \rightarrow+\infty} E_{s q}\left(\varrho_{A_{1} . . A_{m}}^{n}\right)=E_{s q}\left(\varrho_{A_{1} . . A_{m}}\right) .
$$

The same limit relation holds for the function $\hat{E}_{s q}$, since it is lower semicontinuous on the whole space $\mathfrak{S}\left(\mathcal{H}_{A_{1} . . A_{m}}\right)$ and nonincreasing under unilocal operations. This implies the second equality in (48), since $E_{s q}\left(\varrho_{A_{1} . . A_{m}}^{n}\right)=\hat{E}_{s q}\left(\varrho_{A_{1} . . A_{m}}^{n}\right)$ for all $n$ (by the construction of $\hat{E}_{s q}$ ).

Now we are able to prove the first assertion of part B. Indeed, it follows from (47) and (48) that

$$
E_{s q}^{*}\left(\rho_{A_{1} . . A_{m}}^{n}\right) \geq E_{s q}\left(\rho_{A_{1} . . A_{m}}^{n}\right) \geq \widehat{E}_{s q}\left(\rho_{A_{1} . . A_{m}}^{n}\right)
$$

for all $n$ and

$$
E_{s q}^{*}\left(\rho_{A_{1} . . A_{m}}^{0}\right)=E_{s q}\left(\rho_{A_{1} . . A_{m}}^{0}\right)=\widehat{E}_{s q}\left(\rho_{A_{1} . . A_{m}}^{0}\right) .
$$

Thus, the relation (45) and the lower semicontinuity of $\hat{E}_{s q}$ imply (40).

The second assertion of part B follows from the first one and Proposition 3 .

Lemma 4. Let $\rho_{A_{1} . . A_{m} E}$ be a state such that $H\left(\rho_{A_{k}}\right)<+\infty$ for some $k$. Then

$\left|I\left(A_{1}: \ldots: A_{m} \mid E\right)_{\rho}-I\left(A_{1}: \ldots: A_{m} \mid E\right)_{\Phi_{k}^{n} \otimes \operatorname{Id}_{A_{k}^{\mathrm{C}} E}(\rho)}\right| \leq 2\left[H\left(\rho_{A_{k}}\right)-H\left(P_{k}^{n} \rho_{A_{k}} P_{k}^{n}\right)\right]+h_{2}\left(\operatorname{Tr} P_{k}^{n} \rho_{A_{k}}\right)$, where $\Phi_{k}^{n}: A_{k} \rightarrow A_{k}$ is the channel defined in 41) and $A_{k}^{\mathrm{c}}=A_{1} . . A_{m} \backslash A_{k}$.

Proof. By using representation (21) with appropriate permutation of the subsystems we obtain

$$
\left|I\left(A_{1}: \ldots: A_{m} \mid E\right)_{\rho}-I\left(A_{1}: \ldots: A_{m} \mid E\right)_{\Phi_{k}^{n} \otimes \operatorname{Id}_{A_{k}^{c} E}(\rho)}\right|=\left|I\left(A_{k}: A_{k}^{\mathrm{c}} \mid E\right)_{\rho}-I\left(A_{k}: A_{k}^{\mathrm{c}} \mid E\right)_{\Phi_{k}^{n} \otimes \operatorname{Id}_{A_{k}^{\mathrm{c}} E}(\rho)}\right|
$$


Since $\Phi_{k}^{n} \otimes \operatorname{Id}_{A_{k}^{\mathrm{c} E}}(\rho)=P_{k}^{n} \otimes I_{A_{k}^{\mathrm{c} E}} \cdot \rho \cdot P_{k}^{n} \otimes I_{A_{k}^{\mathrm{c} E}}+\tau_{k}^{n} \otimes\left[\operatorname{Tr}_{A_{k}} P_{k}^{n} \otimes I_{A_{k}^{\mathrm{c}} E} \rho\right]$, inequality (23) implies that

$$
I\left(A_{k}: A_{k}^{\mathrm{c}} \mid E\right)_{\Phi_{k}^{n} \otimes \operatorname{Id}_{A_{k}^{\mathrm{c}} E}(\rho)} \geq I\left(A_{k}: A_{k}^{\mathrm{c}} \mid E\right)_{P_{k}^{n} \otimes I_{A_{k}^{\mathrm{c}} E} \cdot \rho \cdot P_{k}^{n} \otimes I_{A_{k}^{\mathrm{c}} E}}-h_{2}\left(\operatorname{Tr} P_{k}^{n} \rho_{A_{k}}\right),
$$

where the vanishing of $I\left(A_{k}: A_{k}^{\mathrm{c}} \mid E\right)$ at the state proportional to $\tau_{k}^{n} \otimes\left[\operatorname{Tr}_{A_{k}} P_{k}^{n} \otimes I_{A_{k}^{\mathrm{c} E}} \rho\right]$ was used 13 Thus, we have

$$
\begin{aligned}
\mid I\left(A_{1}: \ldots:\right. & \left.A_{m} \mid E\right)_{\rho}-I\left(A_{1}: \ldots: A_{m} \mid E\right)_{\Phi_{k}^{n} \otimes \operatorname{Id}_{A_{k}^{\mathrm{c}} E}(\rho)} \mid \leq I\left(A_{k}: A_{k}^{\mathrm{c}} \mid E\right)_{\rho} \\
& -I\left(A_{k}: A_{k}^{\mathrm{c}} \mid E\right)_{P_{k}^{n} \otimes I_{A_{k}^{\mathrm{c}} E} \cdot \rho \cdot P_{k}^{n} \otimes I_{A_{k}^{\mathrm{c} E}}}+h_{2}\left(\operatorname{Tr} P_{k}^{n} \rho_{A_{k}}\right) \\
& \leq 2\left[H\left(\rho_{A_{k}}\right)-H\left(P_{k}^{n} \rho_{A_{k}} P_{k}^{n}\right)\right]+h_{2}\left(\operatorname{Tr} P_{k}^{n} \rho_{A_{k}}\right),
\end{aligned}
$$

where the last inequality follows from Lemma 9 in [30].

Corollary 6. The function $E_{s q}$ defined in (37) is equal to zero on the set of all separable states $\rho_{A_{1} \ldots A_{m}}$ with finite marginal entropies, i.e. such that $H\left(\rho_{A_{k}}\right)<+\infty$ for $k=\overline{1, m}$.

Proof. Let $\left\{P_{1}^{n}\right\} \subset \mathfrak{B}\left(\mathcal{H}_{A_{1}}\right), \ldots,\left\{P_{m}^{n}\right\} \subset \mathfrak{B}\left(\mathcal{H}_{A_{m}}\right)$ be sequences of finite rank projectors strongly converging to the unit operators $I_{A_{1}}, \ldots, I_{A_{m}}$. For each sufficiently large $n$ let $\rho_{A_{1} . . A_{m}}^{n}$ be the state proportional to the operator $P_{1}^{n} \otimes \ldots \otimes P_{m}^{n} \cdot \rho_{A_{1} . . A_{m}} \cdot P_{1}^{n} \otimes \ldots \otimes P_{m}^{n}$. Since $\rho_{A_{1} . . A_{m}}^{n}$ is a finite-dimensional separable state, $E_{s q}\left(\rho_{A_{1} . . A_{m}}^{n}\right)=0$ for all $n$. Since the function $E_{s q}$ is lower semicontinuous on the set $\mathfrak{S}_{*}\left(\mathcal{H}_{A_{1} . . A_{m}}\right)$ defined in (38) by Proposition 5A, we have $E_{s q}\left(\rho_{A_{1} . . A_{m}}\right)=0$.

Since the function $E_{s q}$ is convex and equal to zero on the set of countably-decomposable separable states, i.e. states $\sigma_{A_{1} \ldots A_{m}}$ having the form

$$
\sigma_{A_{1} \ldots A_{m}}=\sum_{i} p_{i} \alpha_{1}^{i} \otimes \ldots \otimes \alpha_{m}^{i}
$$

the assertion of Corollary 6 can be strengthened by saying that $E_{s q}\left(\rho_{A_{1} \ldots A_{m}}\right)=0$ if $\rho_{A_{1} \ldots A_{m}}$ is a mixture of a countably-decomposable separable state and a separable state with finite marginal entropies.

Corollary 4 and Proposition 5 imply the following condition for local continuity of the multipartite squashed entanglement.

Corollary 7. Let $\left\{\rho_{A_{1} \ldots A_{m}}^{n}\right\}$ be a sequence of states converging to a state $\rho_{A_{1} \ldots A_{m}}^{0}$ such that $H\left(\rho_{A_{k}}^{0}\right)$ is finite for $k=\overline{1, m}$. If there exist systems $E_{1}, \ldots E_{m-1}$ and a sequence $\left\{\hat{\rho}_{A_{1} \ldots A_{m} E_{1} \ldots E_{m-1}}^{n}\right\}$ of states converging to a state $\hat{\rho}_{A_{1} \ldots A_{m} E_{1} \ldots E_{m-1}}^{0}$ such that $\hat{\rho}_{A_{1} \ldots A_{m}}^{n}=\rho_{A_{1} \ldots A_{m}}^{n}$ for all $n \geq 0$ and $\lim _{n \rightarrow+\infty} H\left(\rho_{A_{i_{k}} E_{k}}^{n}\right)=H\left(\rho_{A_{i_{k}} E_{k}}\right)<+\infty$ for some set of indexes $i_{1}, \ldots, i_{m-1}$ then relation (40) holds.

By generalizing Example 3 consider arbitrary sequences $\left\{\rho_{A_{1}}^{n}\right\}, \ldots,\left\{\rho_{A_{m}}^{n}\right\}$ and $\left\{\sigma_{A_{1} \ldots A_{m}}^{n}\right\}$ of states converging, respectively, to states $\left\{\rho_{A_{1}}^{0}\right\}, \ldots,\left\{\rho_{A_{m}}^{0}\right\}$ and $\left\{\sigma_{A_{1} \ldots A_{m}}^{0}\right\}$. By obvious

\footnotetext{
${ }^{13}$ We assume that $I\left(A_{k}: A_{k}^{\mathrm{c}} \mid E\right)_{\sigma}=c I\left(A_{k}: A_{k}^{\mathrm{c}} \mid E\right)_{\sigma / c}$ for a positive operator $\sigma$ such that $c=\operatorname{Tr} \sigma>0$.
} 
generalization of the arguments from Example 3 one can apply Corollary 7 to show that

$$
\lim _{n \rightarrow+\infty} E_{s q}\left((1-p) \rho_{A_{1}}^{n} \otimes \ldots \otimes \rho_{A_{m}}^{n}+p \sigma_{A_{1} \ldots A_{m}}^{n}\right)=E_{s q}\left((1-p) \rho_{A_{1}}^{0} \otimes \ldots \otimes \rho_{A_{m}}^{0}+p \sigma_{A_{1} \ldots A_{m}}^{0}\right)
$$

for any $p \in[0,1]$ provided that

$$
\lim _{n \rightarrow+\infty} H\left(\sigma_{A_{k}}^{n}\right)=H\left(\sigma_{A_{k}}^{0}\right)<+\infty \quad \text { and } \quad H\left(\rho_{A_{k}}^{0}\right)<+\infty \quad \text { for } k=\overline{1, m}
$$

regardless of the entropies of the states $\rho_{A_{1}}^{n}, \ldots, \rho_{A_{m}}^{n}$.

The second condition in (50) seems technical (i.e. it can be omitted by using more advanced methods of analysis) but the first condition in (50) is essential. This can be shown by considering the case when $p=1$ and all the states $\sigma_{A_{1} \ldots A_{m}}^{n}$ are pure.

\subsection{On robustness of information gain of quantum measure- ments}

A quantum measurement with finite or countable outcome set $I$ is described mathematically by a quantum instrument $\Upsilon=\left\{\Upsilon_{i}\right\}_{i \in I}$ - collection of quantum operations from a system $A$ to a system $A^{\prime}$ such that $\sum_{i \in I} \operatorname{Tr} \Upsilon_{i}(\rho)=1$ for any state $\rho$ in $\mathfrak{S}\left(\mathcal{H}_{A}\right)$. Many characterictics of a quantum measurement can be expressed via the corresponding Positive Operator Valued Measure (POVM) $\left\{\Upsilon_{i}^{*}\left(I_{\mathcal{H}_{A^{\prime}}}\right)\right\}_{i \in I}$. A quantum measurement is called efficient if all the quantum operations $\Upsilon_{i}$ have Choi rank 1 [16, 25, 38.

Following to Groenewold [12] the information gain of an efficient measurement at a state $\rho$ is defined as

$$
H(\rho)-\sum_{i \in I} p_{i} H\left(\rho_{i}\right)
$$

where $p_{i}=\operatorname{Tr}_{i}(\rho)$ is the probability of the $i$-th outcome and $\rho_{i}=p_{i}^{-1} \Upsilon_{i}(\rho)$ is the corresponding posteriory state. This quantity naturally called the entropy reduction is nonnegative for any efficient measurement [22, 26].

For an arbitrary non-efficient measurement the entropy reduction (51) may be negative and can not be used as its information characteristic. In this case the infromation gain of a quantum measurement described by the instrument $\left\{\Upsilon_{i}\right\}_{i \in I}$ at a state $\rho$ in $\mathfrak{S}\left(\mathcal{H}_{A}\right)$ is given by the quantum mutual information $I(R: E)$ of the state

$$
\sigma_{R E}=\sum_{i \in I} \operatorname{Tr}_{A^{\prime}}\left[\operatorname{Id}_{R} \otimes \Upsilon_{i}\left(\hat{\rho}_{A R}\right)\right] \otimes\left|i_{E}\right\rangle\left\langle i_{E}\right|
$$

where $\hat{\rho}_{A R}$ is a purification of the state $\rho$ and $\left\{\left|i_{E}\right\rangle\right\}$ is a basis in a Hilbert space $\mathcal{H}_{E}$ such that $\operatorname{dim} \mathcal{H}_{E}=\operatorname{card}(I)$ [4]. It is easy to see that for any efficient measurement this quantity coincides with the entropy reduction (51).

Winter showed that the above quantity $I(R: E)_{\sigma}$ has an operational interpretation as the optimal rate at which a measurement gathers information [40]. This result has 
been generalized in 37] to the case when a measurement is applied to part $\mathrm{A}$ of an entangled bipartite state $\rho_{A B}$. It is shown that in this case the optimal rate at which the sender needs to transmit classical information in order to simulate the measurement is equal to the quantum conditional mutual information $I(R: E \mid B)_{\sigma}$ of the state

$$
\sigma_{R E B}=\sum_{i \in I} \operatorname{Tr}_{A^{\prime}}\left[\operatorname{Id}_{R B} \otimes \Upsilon_{i}\left(\hat{\rho}_{A R}\right)\right] \otimes\left|i_{E}\right\rangle\left\langle i_{E}\right|
$$

where $\hat{\rho}_{A R B}$ is a purification of the state $\rho_{A B}$. This quantity is called the information gain in the presence of quantum side information, while the above quantity $I(R: E)_{\sigma}$ - the information gain without quantum side information [2, 5, 37].

In this subsection we analyse continuity properties of the characteristics of quantum measurements described above. Note first that both these characteristics are completely determined by the POVM $\left\{\Upsilon_{i}^{*}\left(I_{\mathcal{H}_{A^{\prime}}}\right)\right\}_{i \in I}$ corresponding to the instrument $\left\{\Upsilon_{i}\right\}_{i \in I}$. Indeed, for a given POVM $\mathbb{M}=\left\{M_{i}\right\}_{i \in I}$ consider the channel

$$
\Psi_{\mathbb{M}}(\rho)=\sum_{i}\left[\operatorname{Tr} M_{i} \rho\right]\left|i_{E}\right\rangle\left\langle i_{E}\right|
$$

from $\mathfrak{S}\left(\mathcal{H}_{A}\right)$ to $\mathfrak{S}\left(\mathcal{H}_{E}\right)$. Then the above defined states $\sigma_{R E}$ and $\sigma_{R E B}$ can be expressed as

$$
\sigma_{R E}=\Psi_{\mathbb{M}_{\Upsilon}} \otimes \operatorname{Id}_{R}\left(\hat{\rho}_{A R}\right) \quad \text { and } \quad \sigma_{R E B}=\Psi_{\mathbb{M}_{\Upsilon}} \otimes \operatorname{Id}_{B R}\left(\hat{\rho}_{A B R}\right)
$$

where $\mathbb{M}_{\Upsilon}=\left\{\Upsilon_{i}^{*}\left(I_{\mathcal{H}_{A^{\prime}}}\right)\right\}_{i \in I}$.

Thus, we may consider the information gain with and without quantum side information as functions of a pair $\left(\mathbb{M}, \rho_{X}\right)$, where $\mathbb{M}$ runs over all POVM on $\mathcal{H}_{A}$ with a given set of outcomes and $\rho_{X}$ runs over the set $\mathfrak{S}\left(\mathcal{H}_{X}\right), X=A, A B$. We will use the notations

$$
\mathrm{IG}\left(\mathbb{M}, \rho_{A}\right)=I(R: E)_{\Psi_{\mathbb{M}} \otimes \operatorname{Id}_{R}\left(\hat{\rho}_{A R}\right)} \quad \text { and } \quad \operatorname{IG}_{\mathrm{QSI}}\left(\mathbb{M}, \rho_{A B}\right)=I(R: E \mid B)_{\Psi_{\mathbb{M}} \otimes \operatorname{Id}_{B R}\left(\hat{\rho}_{A B R}\right)}
$$

where $\hat{\rho}_{A R}$ and $\hat{\rho}_{A B R}$ are purifications of the states $\rho_{A}$ and $\rho_{A B}$ correspondingly.

To obtain continuity conditions in the strongest form we equip the set $\mathfrak{M}_{A}$ of all POVM on $\mathcal{H}_{A}$ with a given outcome set $I$ with the weak convergence. We say that a sequence $\left\{\mathbb{M}_{n}=\left\{M_{i}^{n}\right\}\right\}_{n}$ in $\mathfrak{M}_{A}$ weakly converges to a POVM $\mathbb{M}_{0}=\left\{M_{i}^{0}\right\} \in \mathfrak{M}_{A}$ if the sequence $\left\{M_{i}^{n}\right\}_{n}$ converges to the operator $M_{i}^{0}$ in the weak operator topology for each $i \in I$. The compactness of the unit ball of $\mathfrak{B}\left(\mathcal{H}_{A}\right)$ in the weak operator topology implies that the set $\mathfrak{M}_{A}$ is compact if the set $I$ of outcomes is finite.

We will essentially use the following

Lemma 5. If a sequence $\left\{\mathbb{M}_{n}\right\}$ in $\mathfrak{M}_{A}$ weakly converges to a POVM $\mathbb{M}_{0} \in \mathfrak{M}_{A}$ then the sequence $\left\{\Psi_{\mathbb{M}_{n}}\right\}$ of channels defined in (52) strongly converges to the channel $\Psi_{\mathbb{M}_{0}}$, i.e.

$$
\lim _{n \rightarrow+\infty} \Psi_{\mathbb{M}_{n}}(\rho)=\Psi_{\mathbb{M}_{0}}(\rho) \quad \forall \rho \in \mathfrak{S}\left(\mathcal{H}_{A}\right)
$$


Proof. By Lemma 1 it suffices to show that the sequence $\left\{\Psi_{\mathbb{M}_{n}}(\rho)\right\}$ converges to the state $\Psi_{\mathbb{M}_{0}}(\rho)$ in the weak operator topology for any given state $\rho$. This can be done easily by noting that the weak convergence of the sequence $\left\{\mathbb{M}_{n}\right\}$ to a POVM $\mathbb{M}_{0}$ implies that $\lim _{n \rightarrow+\infty} \operatorname{Tr} M_{i}^{n} \rho=\operatorname{Tr} M_{i}^{0} \rho$ for each $i \in I$.

Now we can prove the following

Proposition 6. A) Let be $\left\{\rho_{A}^{n}\right\}$ be a sequence of states in $\mathfrak{S}\left(\mathcal{H}_{A}\right)$ converging to a state $\rho_{A}^{0}$. If either $\operatorname{card}(I)<+\infty$ or $\lim _{n \rightarrow+\infty} H\left(\rho_{A}^{n}\right)=H\left(\rho_{A}^{0}\right)<+\infty$ then

$$
\lim _{n \rightarrow+\infty} \operatorname{IG}\left(\mathbb{M}_{n}, \rho_{A}^{n}\right)=\operatorname{IG}\left(\mathbb{M}_{0}, \rho_{A}^{0}\right)
$$

for arbitrary sequence $\left\{\mathbb{M}_{n}\right\}$ in $\mathfrak{M}_{A}$ weakly converging to a POVM $\mathbb{M}_{0} \in \mathfrak{M}_{A}$.

B) Let $\left\{\rho_{A B}^{n}\right\}$ be a sequence of states in $\mathfrak{S}\left(\mathcal{H}_{A B}\right)$ converging to a state $\rho_{A B}^{0}$. If either $\operatorname{card}(I)<+\infty$ or $\lim _{n \rightarrow+\infty} H\left(\rho_{X}^{n}\right)=H\left(\rho_{X}^{0}\right)<+\infty$, where $X$ is either $A$ or $A B$, then

$$
\lim _{n \rightarrow+\infty} \operatorname{IG}_{\mathrm{QSI}}\left(\mathbb{M}_{n}, \rho_{A B}^{n}\right)=\mathrm{IG}_{\mathrm{QSI}}\left(\mathbb{M}_{0}, \rho_{A B}^{0}\right)
$$

for arbitrary sequence $\left\{\mathbb{M}_{n}\right\}$ in $\mathfrak{M}_{A}$ weakly converging to a POVM $\mathbb{M}_{0} \in \mathfrak{M}_{A}$.

Proof. Since $\operatorname{dim} \mathcal{H}_{E}=\operatorname{card}(I)$, all the assertions of the proposition follow, by Lemma 5, from Corollary 1 and Proposition 2 ,

Proposition 6 implies, in particular, that for arbitrary sequence $\left\{\mathbb{M}_{n}\right\}$ weakly converging to a POVM $\mathbb{M}_{0}$ we have

$$
\lim _{n \rightarrow+\infty} \operatorname{IG}\left(\mathbb{M}_{n}, \rho_{A}\right)=\operatorname{IG}\left(\mathbb{M}_{0}, \rho_{A}\right)
$$

provided that $H\left(\rho_{A}\right)<+\infty$ and that

$$
\lim _{n \rightarrow+\infty} \operatorname{IG}_{\mathrm{QSI}}\left(\mathbb{M}_{n}, \rho_{A B}\right)=\mathrm{IG}_{\mathrm{QSI}}\left(\mathbb{M}_{0}, \rho_{A B}\right)
$$

provided that either $H\left(\rho_{A B}\right)<+\infty$ or $H\left(\rho_{A}\right)<+\infty$. This property can be treated as stability (robustness) the information gain with and without quantum side information w.r.t. perturbation of quantum measurements.

A sequence of quantum instruments $\Upsilon_{n}=\left\{\Upsilon_{i}^{n}\right\}$ with the same outcomes set $I$ strongly converges to a quantum instrument $\Upsilon_{0}=\left\{\Upsilon_{i}^{0}\right\}$ if

$$
\lim _{n \rightarrow+\infty} \Upsilon_{i}^{n}(\rho)=\Upsilon_{i}^{0}(\rho)
$$

for each $i \in I$ and any state $\rho$ in $\mathfrak{S}\left(\mathcal{H}_{A}\right)$ [28]. It is clear that this convergence implies the weak convergence of the corresponding sequence of POVM $\mathbb{M}_{\Upsilon_{n}}=\left\{\left[\Upsilon_{i}^{n}\right]^{*}\left(I_{A^{\prime}}\right)\right\}$ to the POVM $\mathbb{M}_{\Upsilon_{\Upsilon_{0}}}=\left\{\left[\Upsilon_{i}^{0}\right]^{*}\left(I_{A^{\prime}}\right)\right\}$. Hence, Proposition [6 implies the following

Corollary 8. A) Let $\left\{\rho_{A}^{n}\right\}$ be a sequence of states in $\mathfrak{S}\left(\mathcal{H}_{A}\right)$ converging to a state $\rho_{A}^{0}$. If either $\operatorname{card}(I)<+\infty$ or $\lim _{n \rightarrow+\infty} H\left(\rho_{A}^{n}\right)=H\left(\rho_{A}^{0}\right)<+\infty$ then

$$
\lim _{n \rightarrow+\infty} \operatorname{IG}\left(\mathbb{M}_{\Upsilon_{n}}, \rho_{A}^{n}\right)=\operatorname{IG}\left(\mathbb{M}_{\Upsilon_{0}}, \rho_{A}^{0}\right)
$$


for arbitrary sequence $\left\{\boldsymbol{\Upsilon}_{n}\right\}$ of quantum instruments strongly converging to a quantum instrument $\Upsilon_{0}$.

B) Let $\left\{\rho_{A B}^{n}\right\}$ be a sequence of states in $\mathfrak{S}\left(\mathcal{H}_{A B}\right)$ converging to a state $\rho_{A B}^{0}$. If either $\operatorname{card}(I)<+\infty$ or $\lim _{n \rightarrow+\infty} H\left(\rho_{X}^{n}\right)=H\left(\rho_{X}^{0}\right)<+\infty$, where $X$ is either $A$ or $A B$, then

$$
\lim _{n \rightarrow+\infty} \operatorname{IG}_{\mathrm{QSI}}\left(\mathbb{M}_{\Upsilon_{n}}, \rho_{A B}^{n}\right)=\operatorname{IG}_{\mathrm{QSI}}\left(\mathbb{M}_{\Upsilon_{0}}, \rho_{A B}^{0}\right)
$$

for arbitrary sequence $\left\{\boldsymbol{\Upsilon}_{n}\right\}$ of quantum instruments strongly converging to a quantum instrument $\Upsilon_{0}$.

Corollary 8A agrees with Proposition 1 in [28, since if an instrument $\Upsilon$ is efficient then the information gain $\operatorname{IG}\left(\mathbb{M}_{\Upsilon}, \rho_{A}\right)$ coincides with the entropy reduction (51).

I am grateful to A.S.Holevo and to the participants of his seminar "Quantum probability, statistic, information" (the Steklov Mathematical Institute) for useful discussion.

\section{References}

[1] D.Avis, P.Hayden, I.Savov, "Distributed compression and multiparty squashed entanglement", Journal of Physics A: Mathematical and General, 41(11):115301 (2008); arXiv:0707.2792.

[2] M.Berta, J.M.Renes, M.M.Wilde, "Identifying the information gain of a quantum measurement", IEEE Transactions on Information Theory, 60(12):7987-8006, (2014); arXiv:1301.1594.

[3] F.Buscemi, M.Horodecki, "Towards a unified approach to information-disturbance tradeoffs in quantum measurements", Open Systems and Information Dynamics, 16(01), 29-48 (2009).

[4] F.Buscemi, M.Hayashi, M.Horodecki, "Global information balance in quantum measurements", Physical Review Letters, 100(21):210504 (2008); arXiv:quant-ph/0702166.

[5] F.Buscemi, S.Das, M.M.Wilde, "Approximate reversibility in the context of entropy gain, information gain, and complete positivity", Physical Review A 93:6, 062314 (2016); arXiv:1601.01207.

[6] M.Christandl, A.Winter, "Squashed entanglements - an additive entanglement measure", J. Math. Phys., V.45, 829 (2003).

[7] G.F.Dell'Antonio, "On the limits of sequences of normal states", Commun. Pure Appl. Math. 20, 413-430, 1967.

[8] I.Devetak, J.Yard, "The operational meaning of quantum conditional information", Phys. Rev. Lett. 100, 230501 (2008); arXiv:quant-ph/0612050. 
[9] N.Davis, M.E.Shirokov, M.M.Wilde, "Energy-constrained two-way assisted private and quantum capacities of quantum channels", Phys. Rev. A, 97:6 (2018), 62310 , 31 pp.; arXiv: 1801.08102.

[10] J.Eisert, Ch.Simon, M.B.Plenio "On the quantification of entanglement in infinitedimensional quantum systems" J. Phys. A V.35, N.17, 3911-3923 (2002).

[11] O.Fawzi, R.Renner, "Quantum conditional mutual information and approximate Markov chains", Comm. Math. Phys., 340:2, 575-611 (2015); arXiv:1410.0664.

[12] H.J.Groenewold, "A problem of information gain by quantal measurements", International Journal of Theoretical Physics, 4(5):327-338, 1971.

[13] P.Hayden, R.Jozsa, D.Petz, A.Winter, "Structure of states which satisfy strong subadditivity of quantum entropy with equality", Commun. Math. Phys. 246:2, (2004), 359-374; arXiv:quant-ph/0304007.

[14] F.Herbut "On Mutual Information in Multipartite Quantum States and Equality in Strong Subadditivity of Entropy", J. Phys. A: Math. Gen. 37 (2004) 3535-3542; arXiv:quant-ph/0311193.

[15] A.S.Holevo "Quantum systems, channels, information. A mathematical introduction", Berlin, DeGruyter, 2012.

[16] A.S.Holevo, "Statistical structure of quantum theory", Springer, Berlin 2001.

[17] A.S.Holevo, M.E.Shirokov "On approximation of infinite dimensional quantum channels", Problems of Information Transmission. 2008. V.44. N.2. P.3-22; arXiv: quant-ph/0711.2245.

[18] R.Horodecki, P.Horodecki, M.Horodecki, K.Horodecki, "Quantum entanglement", Rev. Mod. Phys. 81, 865-942 (2009).

[19] A.A.Kuznetsova, "Quantum conditional entropy for infinite-dimensional systems", Theory of Probability and its Applications, 55:4, 709-717 (2011).

[20] D.Leung, G.Smith, "'Continuity of quantum channel capacities", Commun. Math. Phys., 292, 201-215, (2009); arXiv:0810.4931.

[21] E.H.Lieb, M.B.Ruskai, "Proof of the strong suadditivity of quantum mechanical entropy", J.Math.Phys. 14, 1938 (1973).

[22] G.Lindblad, "An entropy inequality for quantum measurement", Comm. Math. Phys. 28, 245 (1972).

[23] G.Lindblad, "Entropy, information and quantum measurements", Comm. Math. Phys. V.33. 305-322 (1973). 
[24] G.Lindblad, "Expectation and Entropy Inequalities for Finite Quantum Systems", Comm. Math. Phys. V.39. N.2. 111-119 (1974).

[25] M.A.Nielsen, I.L.Chuang "Quantum Computation and Quantum Information", Cambridge University Press, 2000.

[26] M.Ozawa, "On information gain by quantum measurement of continuous observable", J. Math. Phys. 27, 759 (1986).

[27] M.B.Plenio, S.Virmani, "An introduction to entanglement measures", Quantum Inf. Comput., V.7 N.1-2, 1-51 (2007); arxiv: quant-ph/0504163.

[28] M.E.Shirokov, "Entropy reduction of quantum measurements", Journal of Mathematical Physics, 52:5 (2011), 052202; arXiv: 1011.3127.

[29] M.E.Shirokov, "Measures of correlations in infinite-dimensional quantum systems", Sbornik: Mathematics, 207:5, 724-768 (2016); arXiv: 1506.06377.

[30] M.E.Shirokov, "Squashed entanglement in infinite dimensions", J. Math. Phys., 57:3 (2016), 32203, 22 pp; arXiv: 1507.08964.

[31] M.E.Shirokov, "Uniform continuity bounds for information characteristics of quantum channels depending on input dimension and on input energy", J. Phys. A, 52:1 (2019), 014001; arXiv:1610.08870.

[32] M.E.Shirokov, "Uniform finite-dimensional approximation of basic capacities of energy-constrained channels", Quant. Inf. Process. (2018) 17: 322. https://doi.org/10.1007/s11128-018-2070-z; arXiv:1707.05641,

[33] M.E.Shirokov, "Strong convergence of quantum channels: continuity of the Stinespring dilation and discontinuity of the unitary dilation", arXiv:1712.03219.

[34] D.Sutter, O.Fawzi, R.Renner "Universal recovery map for approximate Markov chains", Proceedings of the Royal Society A, V.472, N.2186, (2016); arXiv:1504.07251.

[35] R. Tucci, "Entanglement of distillation and conditional mutual information", arXiv: quant-ph/0202144.

[36] A.Wehrl, "General properties of entropy", Rev. Mod. Phys. 50, 221-250, 1978.

[37] M.M.Wilde, P.Hayden, F.Buscemi, M.-H. Hsieh, "The information-theoretic costs of simulating quantum measurements", Journal of Physics A: Mathematical and Theoretical, 45(45):453001, 2012; arXiv:1206.4121.

[38] M.M.Wilde, "Quantum Information Theory", Cambridge, UK: Cambridge Univ. Press, (2013). 
[39] M.M.Wilde"Multipartite quantum correlations and local recoverability", Proceedings of the Royal Society A, 471, N.2177, (2015); arXiv:1412.0333.

[40] A.Winter, "Extrinsic and intrinsic data in quantum measurements: Asymptotic convex decomposition of positive operator valued measures", Commun. Math. Phys., 244(1):157-185, 2004; arXiv:quant-ph/0109050.

[41] D.Yang, K.Horodecki, M.Horodecki, P.Horodecki, J.Oppenheim, W.Song, "Squashed entanglement for multipartite states and entanglement measures based on the mixed convex roof", IEEE Trans. Inf. Theory 55, 3375 (2009), arXiv:0704.2236.

[42] L.Zhang, "Conditional Mutual Information and Commutator", Int. J. Theor. Phys. (2013) 52:21122117. 